\title{
Instability of a periodic flow in geostrophic and hydrostatic balance
}

\author{
Guillaume Simon, Balasubramanya T. Nadiga \\ Computer, Computational and Statistical Sciences Division, Los Alamos National \\ Laboratory, Los Alamos, N.M., U.S.A.
}

\begin{abstract}
Instability of a flow in geostrophic and hydrostatic balance is investigated using numerical simulations of the fully nonlinear, rotating, stratified Boussinesq equations. Burger numbers less than one and small aspect ratio are considered. Although the model we consider has continuous stratification in the vertical, in terms of phenomenology, the large scale baroclinic instability we find is most closely related to that found in the classical setting of Phillips [1]. Indeed, the growth rate and scale of the most unstable mode scale similarly. The advantage of the model we consider lies in being able to use it in studies of unbalanced processes. Preliminary experimentation suggests that there is a small scale instability at small values of Burger number. This instability is initiated in anticyclonic regions, is likely imbalanced, and likely leads to
\end{abstract}

Email addresses: gsimon@lanl.gov (Guillaume Simon), balu@lanl.gov (Balasubramanya T. Nadiga) 
small scale dissipation. By considering two measures of balance- one based on a wave-vortex decomposition and another based on the quasi-geostrophic omega equation - we study the dependence of imbalance on Rossby number. We, however, find that kinetic energy spectra display slopes consistent with quasi-geostrophic turbulence, with no break in slope at high wavenumbers. Keywords: baroclinic instability, quasi-geostrophic turbulence, rotating flows, stratified flows, unbalanced instability

PACS: 92.10ak

\section{Introduction}

Although the ultimate source of energy for flows in the atmosphere and in the oceans can be traced back to the differential solar heating of the polar and tropical latitudes, the process of establishing the large-scale and time-mean circulation in these systems can be complicated and very different. Nevertheless, given the large-scale time-mean circulation in these systems, baroclinic instability may be thought of as the common and crucial link that serves to initiate the process of transfer of energy from large scales to smaller scales. While certain links in the path of energy across scales are well understood, there are others that are not, and identifying and characterizing these latter, poorly understood links is an active area of research. In reality, dissipation 
of energy can only be achieved by molecular friction at small scales, and in particular, in the ocean, routes to dissipation involve phenomena such as waves, tides, flows instabilities and boundary layer processes at intermediate scales. However, given the impossibility of simulating the full range of the phenomena from global-scale circulation to molecular dissipation, only a limited range of scales can be considered in any study; the dominant effects of the excluded range of scales on the resolved scales can only be considered in a necessarily simplified, parameterized form. Needless to mention, this is true of the present study as well and in trying to focus on the baroclinic instability process in a generic form, we disregard a number of realistic features such as tides, boundary effects and the curvature of Earth to mention just a few.

While focussing attention on baroclinic instability, we note that it is commonplace to recognize meanders in the Gulf Stream and Kuroshio as due to baroclinic instability. However, it is not just the fast-flowing western boundary currents that are baroclinically unstable, it is likely that large portions of the world oceans themselves are linearly unstable [2].

From a different point of view, the nature and details of the cascade of energy between slowly evolving large-scale flow and smaller scales are known 
to play a crucial role in determining the nature of predictability in multiscale dynamical systems [3]. Indeed, an increasing number of simulation-based studies are recognizing the importance of correctly describing the non-linear interactions between scales in realizing an accurate prediction $[4,5,6,7,8]$.

In the present study of baroclinic instability, our interest lies specifically in the transfer of energy downscale from the mesoscales to the dissipative scales. To this end, we consider a sinusoidal shear flow in geostrophic balance, baroclinic instability of which will generate the mesoscales. Thereafter, we will describe the main qualitative and quantitative aspects of this flow configuration with an aim of extracting a measure of the unbalanced dynamics. From a geophysical point of view the motivation for being in the regime parameter considered is to get dynamical information of oceanic flows with the horizontal dimension much greater than vertical dimension such as polar and others deep water flows.

We should notice that there exist similar studies, in which the baroclinic flow (Eady like problem [9]) is simplifed in a similar way, and therefore is numerically treated by tri-periodic pseudo-spectral numerical simulation as well. The classic model of Eady [9] of baroclinic instability consider that the motion is on an $f$-plane, that the stratification is uniform, that the motion 
is between two, flat, rigid horizontal surfaces, that the vertical wind shear is uniform and that the flow in is thermal wind balance. Detailled description of how to deal with a Eady like problem in a tri-periodic framework can be found in the Ph.D. dissertation of Simon ([10]) with continuation resulting in two papers ([11], [12]). This way to catch a baroclinic instability was introduced by Salhi \& Cambon ([13]), and addressed in a similar way by Mamatsashvili et al. ([14]) with a very complete analysis based on wave/vortex decomposition and nonmodal stability analysis. The base shear fow was introduced in the abovementioned studies with a constant shear rate $S$ (which is linked, in the present study, to the characteristics of the sinusoid: ratio of the amplitude to the vertical wave length): as an advantage, a spaceuniform (constant) baroclinicity parameter $S f=N^{2}$ where $f$ is the Coriolis frequency and $N$ the Brünt-Wäisäilä frequency. This configuration holds for simpler parametric analysis, related to a constant declination of mean (base) isopycnes with respect to horizontal direction; as a drawback, the numerical procedure is more complicated than the conventional one, due to convection by the mean (base) shear flow (methods for shearing box approximation, by Rogallo [15]).

The rest of the paper is organized as follows: We will first present the 
model and the numerical setup. Then we describe the evolution of the large scales and show some relevant integral quantities. Following this, we examine interaction across scales using spectra and spectral fluxes. Finally we quantify imbalance in the flow. 


\section{A model to study interior dynamics}

Whereas, we are interested in the present study in the internal route to dissipation, the presence of boundaries opens up alternate pathways to dissipation and scale-interaction. For this reason, we choose a configuration without boundaries.

\subsection{Background equations}

The total density field $\rho_{t}$ is partitioned as:

$$
\rho_{t}(\mathbf{x}, t)=\rho_{r}+\rho_{b}(z)+\rho(\mathbf{x}, t)
$$

where $\mathbf{x}=(x, y, z)$ is the vector of space coordinate and $t$ the time coordinate. $\rho_{b}(z)$ is the mean, bulk density profile that is a function of the vertical space coordinate and is obtained by plane-averaging over the $(x, y)$-planes, while $\rho(x, y, z, t)$ is the fluctuating three-dimensional field. Under the assumption that the density fluctuation $\rho$ is small with respect to the bulk density $\rho_{b}$, that the bulk density is small compared the the reference density $\rho_{r}$ : the Boussinesq approximation holds and the density in the inertial term is taken to be constant as $\rho_{r}$. Using the Boussinesq approximation the governing equations for the momentum can be written as follows:

$$
\partial_{t} u_{j}=-u_{k} \partial_{k} u_{j}-\frac{1}{\rho_{r}} \partial_{j} p-\epsilon_{j 3 l} f_{3} u_{l}-g \frac{\rho}{\rho_{r}} \delta_{3 j}+D_{L}\left(u_{j}\right)+D_{S}\left(u_{j}\right)
$$


where $\mathbf{u}$ is the velocity field, $p$ is the pressure field, $g$ is the acceleration of the gravity along $-\mathbf{z}, f$ is the Coriolis frequency along $\mathbf{z}, D_{L}$ is the large scale dissipation operator, $D_{S}$ is the small scale dissipation operator. The dissipation operator will be discussed in sections 2.3 and 2.4. For the sake of simplicity and because it's an acceptable first approximation for ocean dynamics we assume that the density is independent of the salinity. We define the modified density $\theta$ with the dimension of a velocity as:

$$
\theta=\frac{g}{\rho_{r} N} \rho
$$

where $N$ is the Brunt-Väisälä frequency defined, using the state law by:

$$
N=\sqrt{\frac{-g}{\rho_{r}} \partial_{3} \rho_{b}}
$$

The modified density $\theta$ defined by equation 3 is refered in the text as perturbation density. With this change of variables, the complete set of governing equations may be written as

$$
\begin{gathered}
\partial_{i} u_{i}=0 \\
\partial_{t} u_{j}=-u_{k} \partial_{k} u_{j}-\frac{1}{\rho_{r}} \partial_{j} p-\epsilon_{j 3 l} f u_{l}-N \theta \delta_{j 3}+D_{L}\left(u_{j}\right)+D_{S}\left(u_{j}\right) \\
\partial_{t} \theta=-u_{k} \partial_{k} \theta+N u_{3}+D_{L}(\theta)+D_{S}(\theta)
\end{gathered}
$$


In the above equations, $\omega$ is the vorticity field and is defined by:

$$
\omega_{i}=\epsilon_{i j k} \partial_{j} u_{k}
$$

Finally, potential vorticity $q$ is defined as:

$$
q=f \partial_{3} \theta-N \omega_{3}+\omega_{j} \partial_{j} \theta-N f .
$$

\subsection{Geostrophic zonal current}

In order to find an inviscid steady solution, we consider the curl of equation 5b. On using $\Omega$ to represent the vorticity of such a solution, we obtain

$$
0=-U_{j} \partial_{j} \Omega_{i}+\left(\Omega_{j}+f \delta_{j 3}\right) \partial_{j} U_{i}-N \epsilon_{i j 3} \partial_{j} \Theta
$$

Further specializing to a zonal flow with no variation in the $\mathbf{x}$ direction, the $\mathbf{x}$ component of equation 8 reduces to

$$
N \partial_{2} \Theta=\left(\Omega_{2} \partial_{2}+\Omega_{3} \partial_{3}\right) U+f \partial_{3} U
$$

Since vorticity is given by $\Omega_{2}=\partial_{3} U$ and $\Omega_{3}=-\partial_{2} U$ the nonlinear term in equation 9 is zero, leading to the thermal wind relation:

$$
\partial_{2} \Theta=\frac{f}{N} \partial_{3} U
$$

Assuming that the size of the computational domain is $L$ in the horizontal and $H$ in the vertical. For a chosen velocity field as:

$$
U(y, z)=A \sin \left(\frac{2 \pi m}{L} y\right) \sin \left(\frac{2 \pi n z}{H}\right)
$$


using the equation 10 we find density field (equivalently the buoyancy field):

$$
\Theta(y, z)=-\frac{A f L n}{N H m} \cos \left(\frac{2 m \pi}{L} y\right) \cos \left(\frac{2 \pi n z}{H}\right)
$$

A necessary conditions of baroclinic instability can be expressed in term of opposite potential vorticity gradient inside the fluid [16]. It is clear from the visualization that the potential vorticity of the base state change of sign in the domain and satisfy the necessary condition of instability. Because the base flow (equation 11 and 12) is triperiodic we can transform the field variable from equation 5 to 3D Fourier space using:

$$
\left(\begin{array}{l}
u \\
v \\
w \\
\theta
\end{array}\right)(\mathbf{x}, t)=\sum_{\mathbf{k}}\left(\begin{array}{c}
\hat{u} \\
\hat{v} \\
\hat{w} \\
\hat{\theta}
\end{array}\right)(t) e^{\imath \mathbf{k x}}
$$

where $\mathbf{k}$ is the wave vector. In several pictures we also use the mode vector defined by:

$$
\mathbf{m}=\frac{\mathbf{k} L}{2 \pi}
$$




\subsection{Large scale dissipation}

In real geophysical flow there is dissipation of energy at the boundaries. In order keep this mandatory feature in our simulation we use a large scale dissipation operator for modeling the dissipation of the mesoscale in the ocean or synoptic structure in the atmosphere. Even though we are modeling an unforced flow, inverse energy transfer is still active during the decay of the total energy. Without large scale dissipation a condensate of kinetic energy will build up. In order to avoid the formation of a condensate and keep the dynamics as close as possible to ocean turbulence we use large scale dissipation in most of the simulations. Thus by using large scale dissipation, we expect that the modeled dynamics is similar to that of a patch of mesoscale turbulence advected into a zone where the baroclinic forcing is not active anymore. A choice has to be made as to the form of this dissipation operator. We use a Rayleigh damping acting on the mode contained in the plane $k_{z}=0$. This form of the damping has the advantage that it can be expressed in physical space as

$$
D_{L}: u \rightarrow \nu_{L} \int_{0}^{H} u d z
$$




\subsection{Small scale dissipation}

Direct numerical simulation (DNS) of turbulent flows requires a mesh size of the order of the dissipation scale. Therefore, only comparatively low Reynolds numbers can be attained with DNS. However, if we assume that the structure of turbulent flow at submesoscale is independent of the behavior of the dissipative microstructures, we can hope to simulate higher Reynolds numbers provided the statistical effect of subgrid scales on resolved scales is adequately modeled. For simulate microstructures we use a method based on hyperviscosity for the momentum equation and hyperdiffusion for the buoyancy equation. The small scale dissipation operator is defined by:

$$
D_{S}=(-1)^{p+1} \nu_{S}\left(\nabla_{h}^{2}+\left(\frac{\Delta_{z}}{\Delta_{h}}\right)^{\frac{6 p-2}{3 p}} \nabla_{z}^{2}\right)^{p}
$$

where $\Delta_{h}$ is the horizontal mesh size, $\Delta_{z}$ is the vertical mesh size, $p$ is the exponent, $\nu_{S}$ is the hyperviscous coefficient. We take the hyperdiffusion coefficient to be equal to the hyperviscous coefficient. In spectral space the dissipation operator is given by:

$$
\hat{D}_{S}=\nu_{S}\left(\mathbf{k}_{h}^{2}+\left(\frac{\Delta_{z}}{\Delta_{h}}\right)^{\frac{6 p-2}{3 p}} k_{z}^{2}\right)^{p}
$$

where $\mathbf{k}_{h}$ is the horizontal wave number and $k_{z}$ the vertical wave number. 


\subsection{Numerical setup}

We use the Sandia-LANL pseudo-spectral code documented in Taylor et al. [17] and in Kurien and Taylor [18]. The hyperviscous and hyperdiffusion exponent $p$ in 16 is set to 4 . We fix the ratio of the vertical to horizontal size of the domain $H / L$ at 0.004 . For a fixed aspect ratio, the control parameters are the Brunt-Väisälä frequency $(N)$, the Coriolis frequency $(f)$, the amplitude of the sinusoid $(A)$, the horizontal periodicity of the sinusoid $(m)$, the vertical periodicity of the sinusoid $(n)$, the small scale dissipation coefficient $\left(\nu_{S}\right)$ and the large scale dissipation coefficient $\left(\nu_{L}\right)$.

The Rossby radius is an estimate of length of the most energetic scale in geostrophic turbulence and is defined by:

$$
L_{R}=\frac{N H}{n f}
$$

The zonal length of the linearly most unstable baroclinic wave is of the order of the Rossby radius:

$$
L_{I}=C_{I}^{L} L_{R}
$$

where $C_{I}^{L}$ is a constant of $O(1)$. We define the Burger number as:

$$
B u=\frac{N H}{n f L}=\frac{L_{R}}{L}
$$


Further, in order that the instability scale is contained in the domain, we choose $0.05 \leq B u \leq 0.5$ (see table 1$)$.

We chose the hyper-viscosity coefficient as small as possible while keeping the peak in the dissipation spectrum below the truncation wave-number. We require that $\frac{\Delta_{h}}{\eta}<0.3$, where $\eta$, the hyper-dissipative scale is defined as:

$$
\eta=2 \pi\left(\nu_{S}^{3} / \epsilon_{S}^{1 / 2}\right)^{1 /(3 p-1)}
$$

As for small scale dissipation, the hyperviscous Reynolds number is defined as:

$$
R e_{S}=\frac{A L^{2 p-1}}{\nu_{S}} .
$$

The hyperviscous Reynolds number for the case simulated in this study is taken between $6.310^{11}$ and $1.810^{19}$ (see table 1).

A non-dimensional analysis of the system 5 shows that the large scale dissipation can be characterized by the non-dimensional number:

$$
R e_{L}=\frac{A}{\nu_{L} H L}
$$

The large scale dissipation number is taken between 0.9 and $\infty$ (see table 1) and referred to as the large scale Reynolds number.

The Rossby number of the base flow is defined by:

$$
R o=\frac{A}{L f}
$$


When the Rossby number is infinitely small the dynamics is said as balanced and can be predicted by quasi-geostrophic theory. The balanced dynamic is assimilated as the slow manifold. For small but finite Rossby number the slow manifold can't exist without the presence of a fast manifold. The fast manifold categorizes unbalanced motions. Among the quasigeostrophic model and the Boussinesq model, there are variants of higher-order balance theories for a finite Rossby number. Each model include more or less of the fast manifold or is more or less accurate in describing the dynamics of the flow. A review of this intermediate balanced model can be found in [19].

In our study, the Rossby of the base flow is taken between $3.510^{-3}$ and $710^{-5}$. Inertial instability is an instability that leads to horizontal accelerations away from an equilibrium position when parcels are perturbed horizontally. The instability analysis involves a cross-wise displacement of a tube of parcels aligned with the current, and then an evaluation of the Coriolis force and horizontal pressure gradient force on the parcels at their new location. We note that the initial velocity is too weak to allow inertial instability: The necessary condition of inertial instability is:

$$
f(f-2 \pi m / L \cos (2 \pi m y / L) \sin (2 \pi n z / H))<0
$$

The necessary condition of inertial instability below (see Holton [20]) is not 
satisfied.

In the study of baroclinic instability, an useful non-dimensional number is the Richardson number:

$$
R i=\frac{N}{S}=\frac{N H}{4 n A}
$$

where $S$ is the characteristic vertical shear of the sinusoidal

$$
S=\frac{n 4 A}{H}
$$

The characteristic Richardson number must be big enough in order for the baroclinic instability to be dominant. The value of this characteristic Richardson number for the runs presented in this study is between 3 and 337 (see table 2).

In general each parameter will influences the result of the simulation. For large Burger number $(B u)$ the flow will tend to act like a strongly stratified flown and for small Burger number the flow will act more like a strongly rotating flow. For small Rossby $(R o)$ number the flows are strongly influenced by the rotation and the anisotropic turbulence is organized in vertical column wise structure. For large Froude $(F r)$ number the flow will have the tendency to organize himself in layered like structure. $m$ is the horizontal periodicity of the sinusoid and will setup the number of structure that we 
show in the $y$ direction. The $n$ parameter will act on the time developpement of the baroclinic instability: increasing $n$ decrease the time when appeart the baroclinic instability. The $R e$ number will be characteristics of the number of non-linear interaction present in the flow. The bigger the Reynolds number the more non-linear interaction. 


\begin{tabular}{||c||c|c|c|c|c|c|c|c||}
\hline \hline Run n $^{\circ}$ & $B u$ & $R o$ & $m$ & $n$ & $R e_{L}$ & $R e_{S}$ & $N h$ & $N z$ \\
\hline \hline Run 1 & 0.05 & $710^{-4}$ & 1 & 1 & 9.4 & $1.610^{+18}$ & 256 & 32 \\
\hline Run 2 & 0.05 & $710^{-4}$ & 1 & 2 & 9.4 & $2.610^{+18}$ & 256 & 32 \\
\hline Run 3 & 0.1 & $710^{-5}$ & 1 & 1 & 9.4 & $1.810^{+19}$ & 256 & 32 \\
\hline Run 4 & 0.1 & $1.2510^{-4}$ & 1 & 1 & 9.4 & $1.810^{+19}$ & 256 & 32 \\
\hline Run 5 & 0.1 & $2.510^{-4}$ & 1 & 1 & 9.4 & $1.810^{+19}$ & 256 & 32 \\
\hline Run 6 & 0.1 & $710^{-4}$ & 1 & 1 & 0.94 & $2.610^{+18}$ & 256 & 32 \\
\hline Run 7 & 0.1 & $710^{-4}$ & 1 & 1 & 9.4 & $6.310^{+11}$ & 32 & 32 \\
\hline Run 8 & 0.1 & $710^{-4}$ & 1 & 1 & 9.4 & $2.010^{+13}$ & 64 & 32 \\
\hline Run 9 & 0.1 & $710^{-4}$ & 1 & 1 & 9.4 & $1.610^{+16}$ & 128 & 32 \\
\hline Run 10 & 0.1 & $710^{-4}$ & 1 & 1 & 9.4 & $2.110^{+18}$ & 256 & 16 \\
\hline Run 11 & 0.1 & $710^{-4}$ & 1 & 1 & 9.4 & $2.110^{+18}$ & 256 & 32 \\
\hline Run 12 & 0.1 & $710^{-4}$ & 1 & 1 & 9.4 & $2.110^{+18}$ & 256 & 64 \\
\hline Run 13 & 0.1 & $710^{-4}$ & 1 & 1 & 9.4 & $2.610^{+18}$ & 256 & 32 \\
\hline Run 14 & 0.1 & $710^{-4}$ & 1 & 1 & 9.4 & $1.810^{+19}$ & 256 & 32 \\
\hline Run 15 & 0.1 & $710^{-4}$ & 1 & 1 & 93.7 & $2.610^{+18}$ & 256 & 32 \\
\hline Run 16 & 0.1 & $710^{-4}$ & 1 & 1 & 937 & $2.610^{+18}$ & 256 & 32 \\
\hline Run 17 & 0.1 & $710^{-4}$ & 1 & 1 & $\infty$ & $2.110^{+18}$ & 256 & 32 \\
\hline Run 18 & 0.1 & $710^{-4}$ & 2 & 1 & 9.4 & $2.610^{+18}$ & 256 & 32 \\
\hline Run 19 & 0.1 & $1.510^{-3}$ & 1 & 1 & 19 & $5.210^{+18}$ & 256 & 32 \\
\hline Run 20 & 0.1 & $2.510^{-3}$ & 1 & 1 & 9.4 & $1.910^{+19}$ & 256 & 32 \\
\hline Run 21 & 0.1 & $710^{-3}$ & 1 & 1 & 9.4 & $1.810^{+19}$ & 256 & 32 \\
\hline Run 22 & 0.15 & $710^{-4}$ & 1 & 1 & 9.4 & $2.610^{+18}$ & 256 & 32 \\
\hline Run 23 & 0.2 & $710^{-4}$ & 1 & 1 & 9.4 & $2.610^{+18}$ & 256 & 32 \\
\hline Run 24 & 0.25 & $710^{-4}$ & 1 & 1 & 9.4 & $2.610^{+18}$ & 256 & 32 \\
\hline Run 25 & 0.25 & $3.510^{-3}$ & 1 & 1 & 46 & $1.310^{+19}$ & 256 & 32 \\
\hline Run 26 & 0.3 & $710^{-4}$ & 1 & 1 & 9.4 & $2.610^{+18}$ & 256 & 32 \\
\hline Run 27 & 0.35 & $710^{-4}$ & 1 & 1 & 9.4 & $2.610^{+18}$ & 256 & 32 \\
\hline Run 28 & 0.4 & $710^{-4}$ & 1 & 1 & 9.4 & $2.610^{+18}$ & 256 & 32 \\
\hline Run 29 & 0.45 & $710^{-4}$ & 1 & 1 & 9.4 & $2.610^{+18}$ & 256 & 32 \\
\hline \hline
\end{tabular}

Table 1: Non-dimensional numbers in simulations performed ordered by growing $B u$. Ro, $m, n, R_{L}, R e_{S}, N h$ and $N z . B u$ is the Burger number defined by equation 20. Ro is the Rossby number defined by equation 24 . $m$ is the meridional periodicity appearing in equation 11 and $12 . n$ is the vertical periodicity appearing in equation 11 and $12 . R e_{L}$ is the large scale Reynolds number defined by $23 . R e_{S}$ is the small scale Reynolds number defined by equation 22. $N h$ is the horizontal number of grid points. $N z$ is the vertical number of grid points. 19 


\begin{tabular}{||c||c|c|c||}
\hline \hline Run $^{\circ}$ & $F r$ & $R i$ & $\max \left(\Delta_{h} / \eta\right)$ \\
\hline \hline Run 1 & $2.610^{-7}$ & 16 & 0.21 \\
\hline Run 2 & $2.610^{-7}$ & 16 & 0.20 \\
\hline Run 3 & $1.110^{-7}$ & 337 & 0.21 \\
\hline Run 4 & $210^{-8}$ & 200 & 0.20 \\
\hline Run 5 & $410^{-8}$ & 100 & 0.22 \\
\hline Run 6 & $1.110^{-7}$ & 33 & 0.17 \\
\hline Run 7 & $1.110^{-7}$ & 33 & 0.17 \\
\hline Run 8 & $1.110^{-7}$ & 33 & 0.17 \\
\hline Run 9 & $1.110^{-7}$ & 33 & 0.20 \\
\hline Run 10 & $1.110^{-7}$ & 33 & 0.19 \\
\hline Run 11 & $1.110^{-7}$ & 33 & 0.17 \\
\hline Run 12 & $1.110^{-7}$ & 33 & 0.18 \\
\hline Run 13 & $1.110^{-7}$ & 33 & 0.17 \\
\hline Run 14 & $1.110^{-7}$ & 33 & 0.21 \\
\hline Run 15 & $1.110^{-7}$ & 33 & 0.17 \\
\hline Run 16 & $1.110^{-7}$ & 33 & 0.17 \\
\hline Run 17 & $1.110^{-7}$ & 33 & 0.17 \\
\hline Run 18 & $1.110^{-7}$ & 33 & 0.17 \\
\hline Run 19 & $2.310^{-7}$ & 16 & 0.18 \\
\hline Run 20 & $410^{-7}$ & 10 & 0.28 \\
\hline Run 21 & $1.110^{-7}$ & 3 & 0.25 \\
\hline Run 22 & $7.910^{-8}$ & 50 & 0.15 \\
\hline Run 23 & $5.910^{-8}$ & 67 & 0.14 \\
\hline Run 24 & $4.710^{-8}$ & 84 & 0.13 \\
\hline Run 25 & $2.310^{-7}$ & 16 & 0.17 \\
\hline Run 26 & $3.910^{-8}$ & 101 & 0.14 \\
\hline Run 27 & $3.310^{-8}$ & 118 & 0.14 \\
\hline Run 28 & $2.910^{-8}$ & 135 & 0.14 \\
\hline Run 29 & $2.610^{-8}$ & 152 & 0.14 \\
\hline \hline & & & \\
\hline
\end{tabular}

Table 2: Additional non-dimensional parameters in simulations considered. $R i$ is the characteristic Richardson number of the base flow defined by equation 26. $\eta$ is the small scale dissipation defined by equation 21. $\Delta_{h}$ is the horizontal grid size. $\mathrm{Fr}$ is the Froude number defined by $\mathrm{Fr}=\frac{A}{H N}$. 


\section{Large scale flow evolution}

\subsection{Geometry of the instability}

We consider a characteristic time of the base state $\tau_{B}$ defined as

$$
\tau_{B}=\frac{N}{S f}
$$

In the Charney problem [21] or the Eady problem [9] this time $\tau_{B}$ is related to the growth rate of the baroclinic instability (see Pedlosky [22] for a classical review and Salhi \& Cambon [13] for the Rapid Distortion Theory). We add a small perturbation of velocity $\mathbf{u}_{p}$ and density $\theta_{p}$ to the base state as

$$
\begin{gathered}
\mathbf{u}_{p}=\epsilon \mathbb{P}(\gamma(\mathbf{x})) \\
\theta_{p}=\epsilon \zeta(\mathbf{x})
\end{gathered}
$$

Where $\epsilon$ is a control parameter for the amplitude of the perturbation, $\gamma(\mathbf{x})$ is a random vector $[0,1]^{3}$ and $\zeta(\mathbf{x})$ is a random number in $[0,1] . \mathbb{P}$ is the projection operator for assuring that the velocity perturbation is incompressible. The small perturbation superimposed on the unstable geostrophic initial flow develops into baroclinic waves (see figures 1 and $2 a$ ) that later connect to each

other (see figures 1). On each horizontal plane the phase line of the wave is inclined. The degree of asymmetry vary with different $B u$. The smaller $B u$ 
is greater is the asymmetry. Assuming that the sense of ambient rotation is as in the Northern hemisphere, the phase lines are inclined from NorthWest to South-East. While $v$ and $\omega_{3}$ display a continuous pattern of upshear phase tilting (see McWilliams [23]), the phase tilting in $w$ is more discontinuous. We note that $\theta, q, \omega_{1}, \omega_{2}$ exhibit discontinuous downshear phase titling. As non-linearity increases the baroclinic waves start to meander and an asymmetry develops between cyclones and anti-cyclones (see figure 2b).

Soon thereafter, the baroclinic waves breaks into eddies and turbulence ensues with intense cyclones (see figure 2c). Inverse cascade of energy then leads to accumulation of energy in the large scales both in the horizontal and in the vertical. 2d. However, given the form of the large scale dissipation, the barotropic energy is dissipated and the remnant baroclinicity is seen to peak at the second mode. 


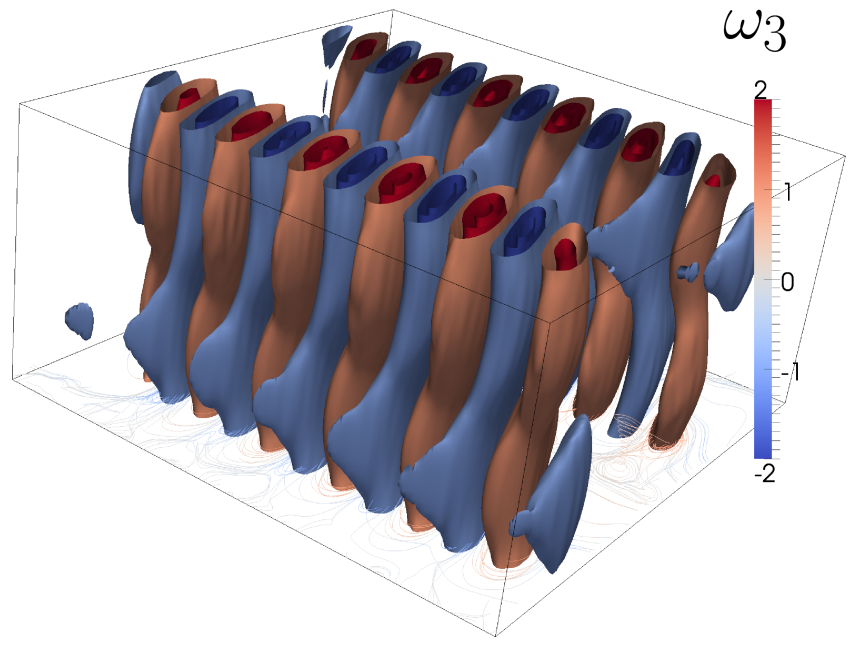

(a) Run 19 in table 1 at $t / \tau_{B}=4.6(B u=0.1)$

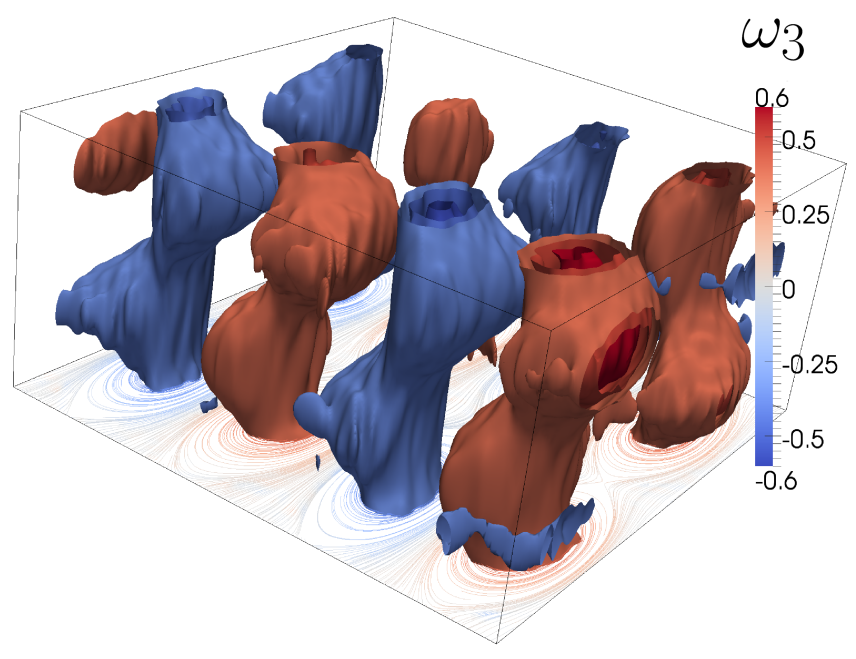

(b) Run 26 in table 1 at $t / \tau_{B}=4.3(B u=0.3)$

Figure 1: Isosurface of vertical vorticity and path line colored by vertical vorticity. 


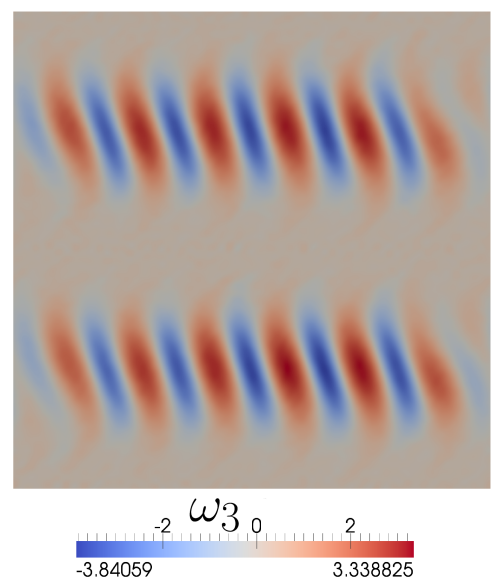

(a) $t / \tau_{B}=4.6$

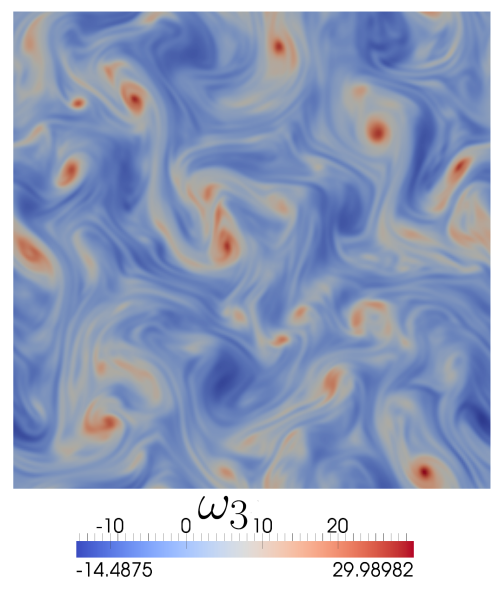

(c) $t / \tau_{B}=11.1$

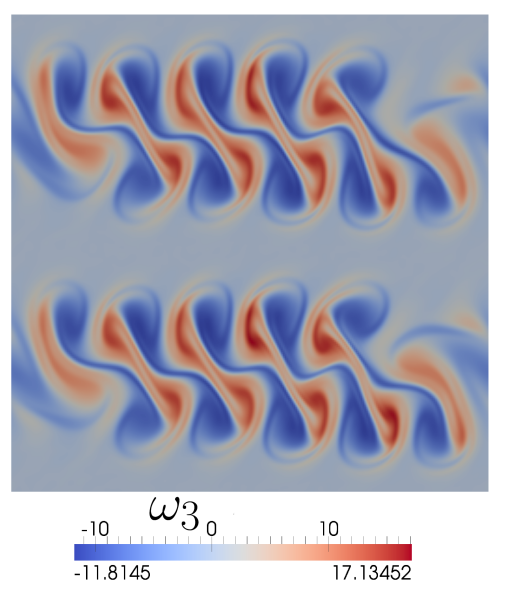

(b) $t / \tau_{B}=6.5$

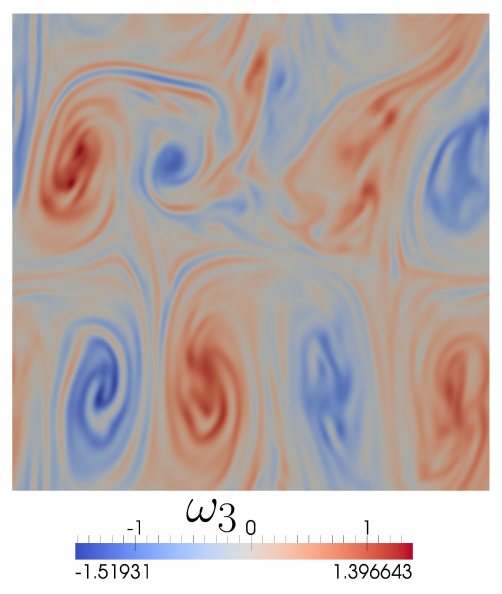

(d) $t / \tau_{B}=148.7$

Figure 2: Vertical vorticity field on the plane $z=H / 2$ for run 19 in table 1 . 


\subsection{Energy Evolution}

Kinetic and potential energy of the base state (defined by equation 11 and equation 12) is given by:

$$
\begin{gathered}
E k_{B}=\frac{A^{2}}{8} m^{2} s^{-2} \\
E p_{B}=\frac{A^{2}}{8} \frac{f^{2} L^{2}}{H^{2} N^{2}} m^{2} s^{-2} .
\end{gathered}
$$

The ratio of available potential to kinetic energy of the base state is:

$$
\alpha_{E p_{B} / E k_{B}}=\frac{f^{2} L^{2}}{H^{2} N^{2}}
$$

and this ratio also happens to be the inverse of the our unconventional Burger number squared or a conventional Burger number. In the simulations considered,

$$
2 \leq \alpha_{E p_{B} / E k_{B}} \leq 20
$$

somewhat comparable to those of the mesoscales in the ocean. The sum of the kinetic and potential energy:

$$
E m(t)=E p(t)+E k(t)
$$

which we call mechanical energy is conserved for a non dissipative flow:

$$
\partial_{t} E m=-\epsilon_{m}
$$


where $\epsilon_{m}$ is the total dissipation. The equations for the kinetic energy and the potential energy involve buoyancy flux $P_{w \theta}$ as the conversion term:

$$
\begin{gathered}
\partial_{t} E p=P_{w \theta}-\epsilon_{p} \\
\partial_{t} E k=-P_{w \theta}-\epsilon_{k}
\end{gathered}
$$

For all the runs dominated by baroclinic instability $(R i>1$ and large enough $)$ the evolution of kinetic, potential, and mechanical energy looks like that in figure 3. Potential energy decays because of baroclinic instability while kinetic energy grows during the instability and then decays. We note that at larger Burger numbers, after saturation, kinetic energy displays an extended period of weak dissipation before ultimately decaying. For larger $B u$ there is a self-sustaining oscillation of the baroclinic structure. 


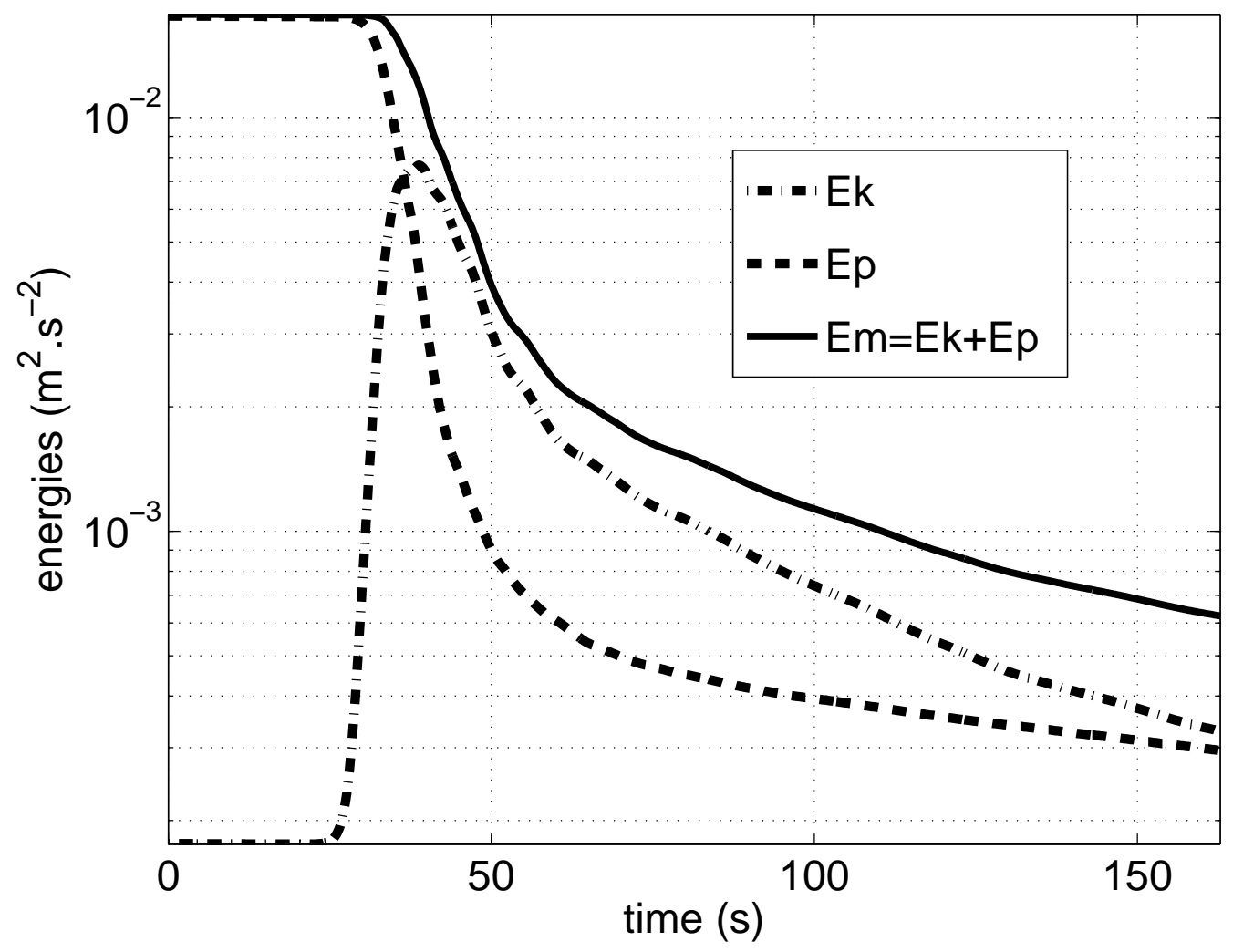

Figure 3: Energy evolution for run 13 in table 1. Ek is the kinetic energy appearing in equation $35 \mathrm{~b} . E p$ is the potential energy appearing in equation 35a. Em is the mechanical energy appearing in equation 34 


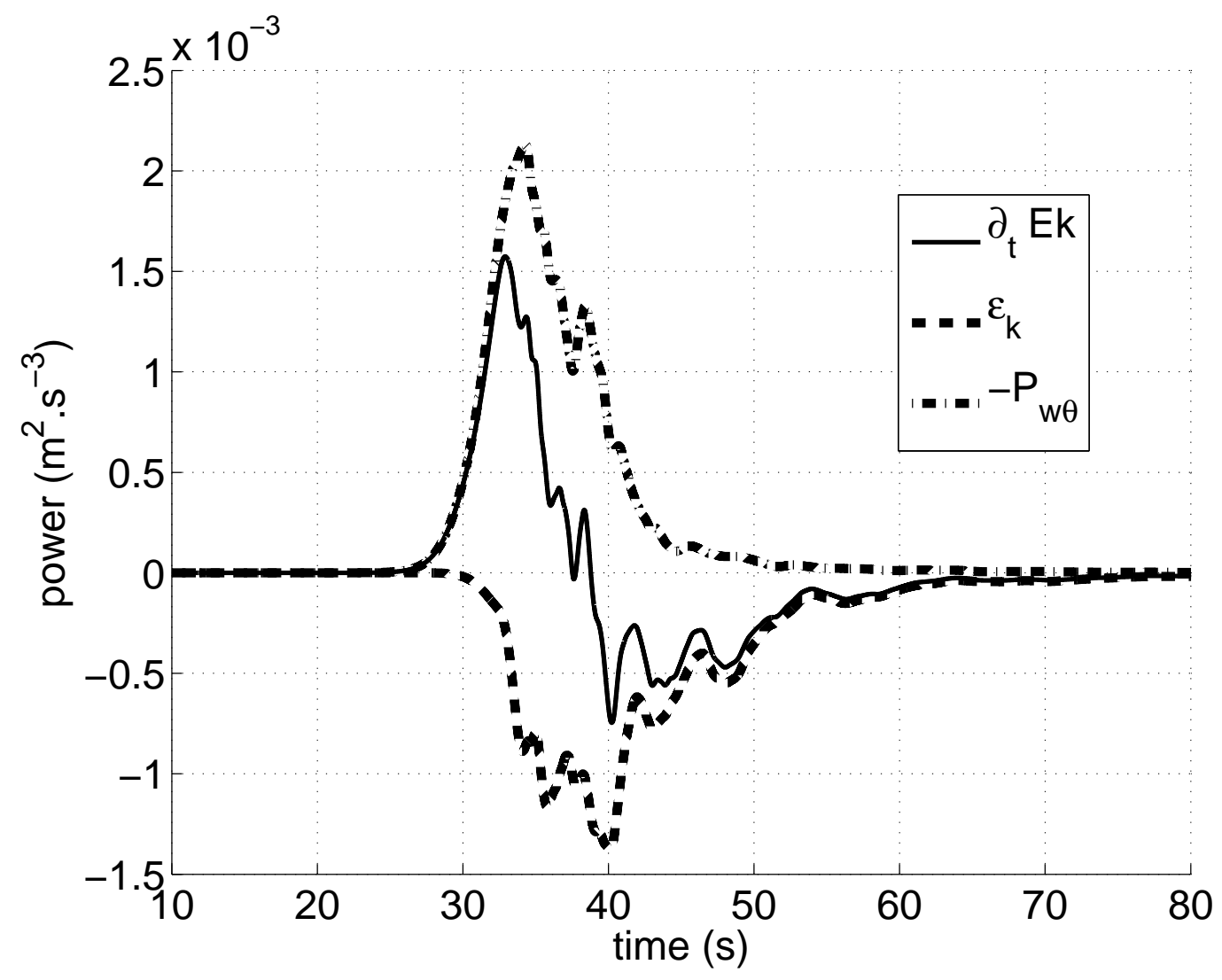

Figure 4: Balance of kinetic energy for run 13 in table 1. Ek is the kinetic energy. $P_{w \theta}$ is the perturbation density flux. $\epsilon_{k}$ is the total dissipation of kinetic energy. 


\subsection{Dissipation}

The development of the mesoscale instability coincides with an increase in large scale dissipation (see figure 5). However, we note that large scale dissipation is chosen to act only on the barotropic mode, whereas, dissipation in the buoyancy equation acts only on perturbation density which is necessarily baroclinic. For this reason, large scale dissipation of potential energy is negligible. Thus large scale dissipation acts to dissipate kinetic energy in as much as the inverse cascade of energy pumps energy into the barotropic mode. As for small scale dissipation, it is our hypothesis that it will be enhanced over the period of baroclinic instability. But as we will see in section 5, the peak of small scale dissipation is not (temporally) coincident with the peak of kinetic energy suggesting that imbalance is playing a role in dissipation. 


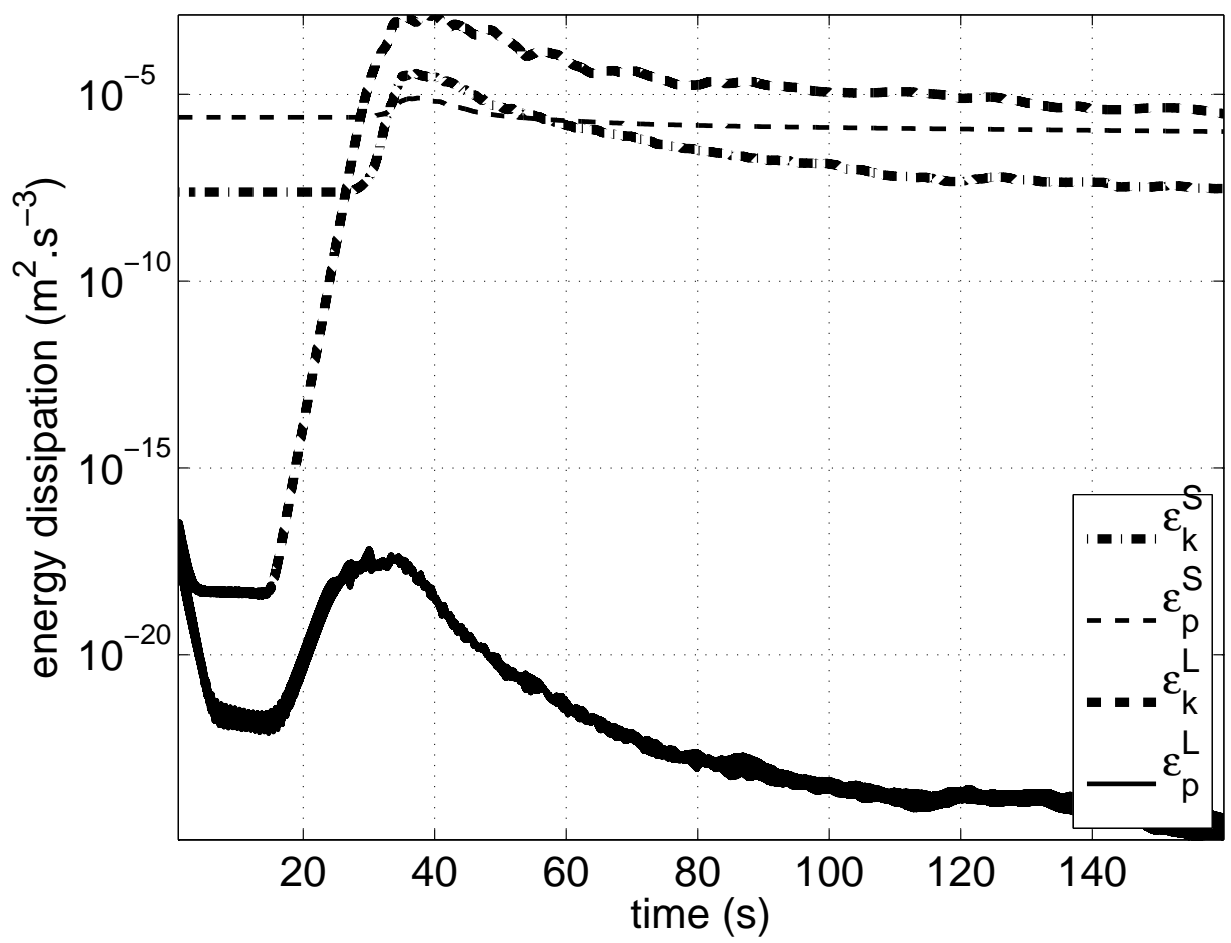

Figure 5: Energy dissipation as function of time for run 13 in table 1. $\epsilon_{p}^{S}$ is the small scale dissipation of potential energy, $\epsilon_{p}^{L}$ is the large scale dissipation of potential energy and $\epsilon_{P}=\epsilon_{p}^{L}+\epsilon_{p}^{S}$ is the dissipation term in potential energy equation $35 \mathrm{a} . \epsilon_{k}^{S}$ is the small scale dissipation of kinetic energy, $\epsilon_{k}^{L}$ is the large scale dissipation of kinetic energy and $\epsilon_{k}=\epsilon_{k}^{L}+\epsilon_{k}^{S}$ is the dissipation term the kinetic energy equation $35 \mathrm{~b}$. 


\section{Spectral characteristics of the large scale instability}

We partition evolution of the flow into three periods for purposes of evaluating spectral properties: a linear growth phase, a non-linear growth and saturation phase and a decay phase. We mainly focus on the non-linear growth and saturation phase wherein baroclinic dynamics are dominant.

\subsection{Spectral flux and balance of energy}

To understand transfer of energy across scale, we use the equation for the spectral balance of kinetic and potential energy:

$$
\begin{aligned}
& \partial_{t} \hat{E} p(\mathbf{k})=\hat{T} p(\mathbf{k})+\hat{P}_{w \theta}(\mathbf{k})-\hat{\epsilon}_{p}(\mathbf{k}) \\
& \partial_{t} \hat{E} k(\mathbf{k})=\hat{T} k(\mathbf{k})-\hat{P}_{w \theta}(\mathbf{k})-\hat{\epsilon}_{k}(\mathbf{k})
\end{aligned}
$$

where $\hat{E} k$ is the kinetic energy spectral density and is defined:

$$
\hat{E} k(\mathbf{k})=\frac{1}{2} \hat{u}_{i}(\mathbf{k}) \hat{u}_{i}(\mathbf{k})^{*}
$$

$\hat{E} p$ is the potential energy spectral density and is defined as:

$$
\hat{E} p(\mathbf{k})=\frac{1}{2} \hat{\theta}(\mathbf{k}) \hat{\theta}(\mathbf{k})^{*}
$$

$\hat{P}_{w \theta}$ is the spectra of the vertical flux of perturbation density, $\hat{\epsilon}_{p}$ is the dissipation spectra of potential energy and and $\hat{\epsilon}_{k}$ is the dissipation spectra of 
kinetic energy. The non-linear potential energy transfer $\hat{T}_{p}$ and the non-linear kinetic energy transfer $\hat{T}_{k}$ are defined by:

$$
\begin{gathered}
\hat{T} p(\mathbf{k})=-\frac{1}{2} \imath k_{i}\left(\hat{\theta}(\mathbf{k}) \widehat{u_{i} \theta}(\mathbf{k})^{*}-\hat{\theta}(\mathbf{k})^{*} \widehat{u_{i} \theta}(\mathbf{k})\right) \\
\hat{T} k(\mathbf{k})=-\frac{1}{2} \imath k_{i}\left(\hat{u}_{j}(\mathbf{k}) \widehat{u_{i} u_{j}}(\mathbf{k})^{*}-\hat{u}_{j}(\mathbf{k})^{*} \widehat{u_{i} u_{j}}(\mathbf{k})\right)
\end{gathered}
$$

The non-linear growth and saturation phase is characterized by a balance between perturbation density flux and non-linear flux at scales between that of the system and roughly twice that of the instability. However, while the nonlinear flux cascades potential energy downscale, it cascades kinetic energy upscale (see thick solid line for $\hat{T} p$ and $\hat{T} k$, thick dashed line for $\hat{P}_{w \theta}$ on figures 6). All the energy of the dynamic comes from the availlable potential energy of the zonal flow (see tick solides curves on 6), and the transfers are consistent with that expected for the nonlinear phase of baroclinic instability and geostrophic turbulence. Dissipation of large scale kinetic energy is seen to occur only at the very low wave numbers. For reference, figure 7 shows the downscale transfer of energy and the associated small scale dissipation for different Burger numbers. This transfer is mostly active in the saturation phase and transfers a small amount of energy from the scale of the baroclinic instability to the small scale dissipation scale. The downscale transfer of kinetic energy is different for different $B u$ because the horizontal scale of 
the baroclinic instability change wiht $B u$. There is a spike on the plot for $B u=0.2$ because of the not perfect averaging method. 


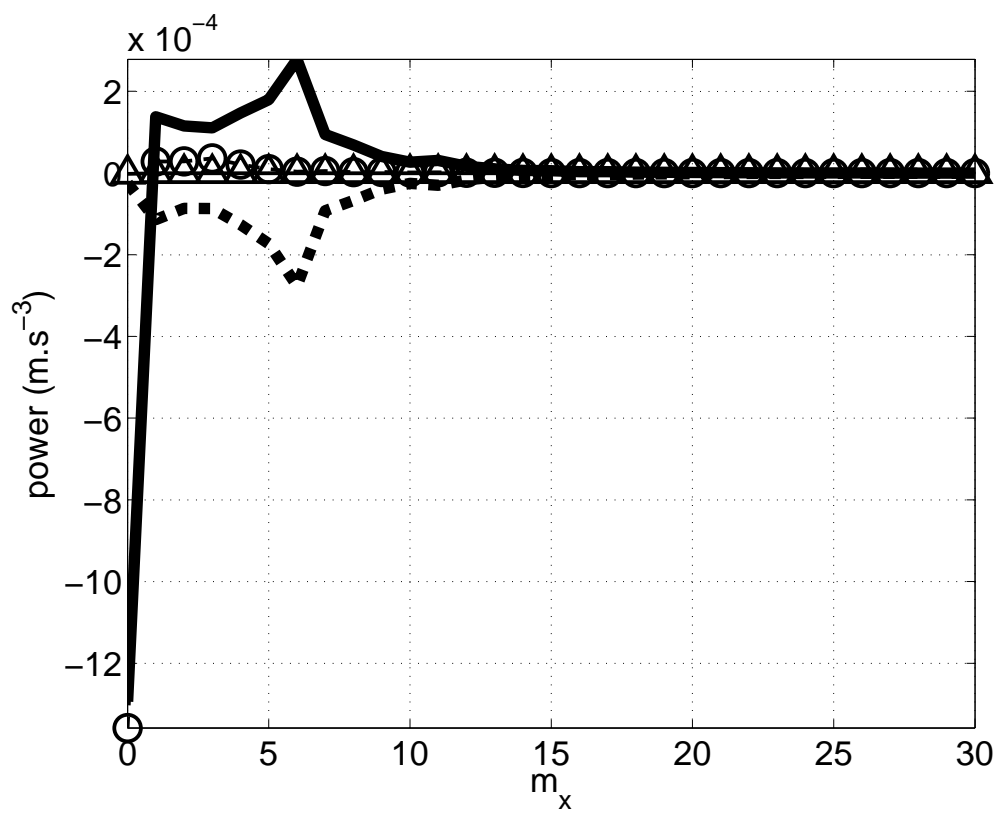

(a) Ep balance: equation 36a

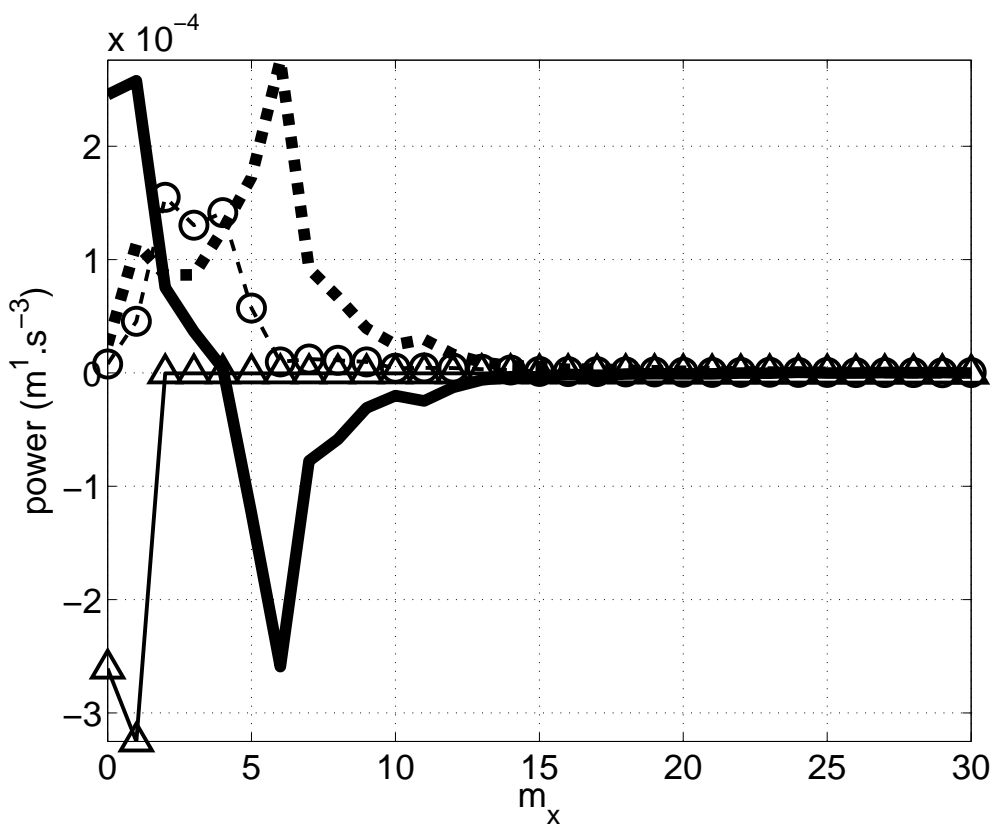

(b) $E k$ balance: equation $36 \mathrm{~b}$

Figure 6: Saturation phase for run 13 in table 1. Thick solid line: non-linear transfer; fine dashed line with circle: acceleration; fine solid line with triangle: dissipation; thick dashed line: buoyancy flux. Averaged from $28 s$ to $40 s$ with a sampling time of 341 


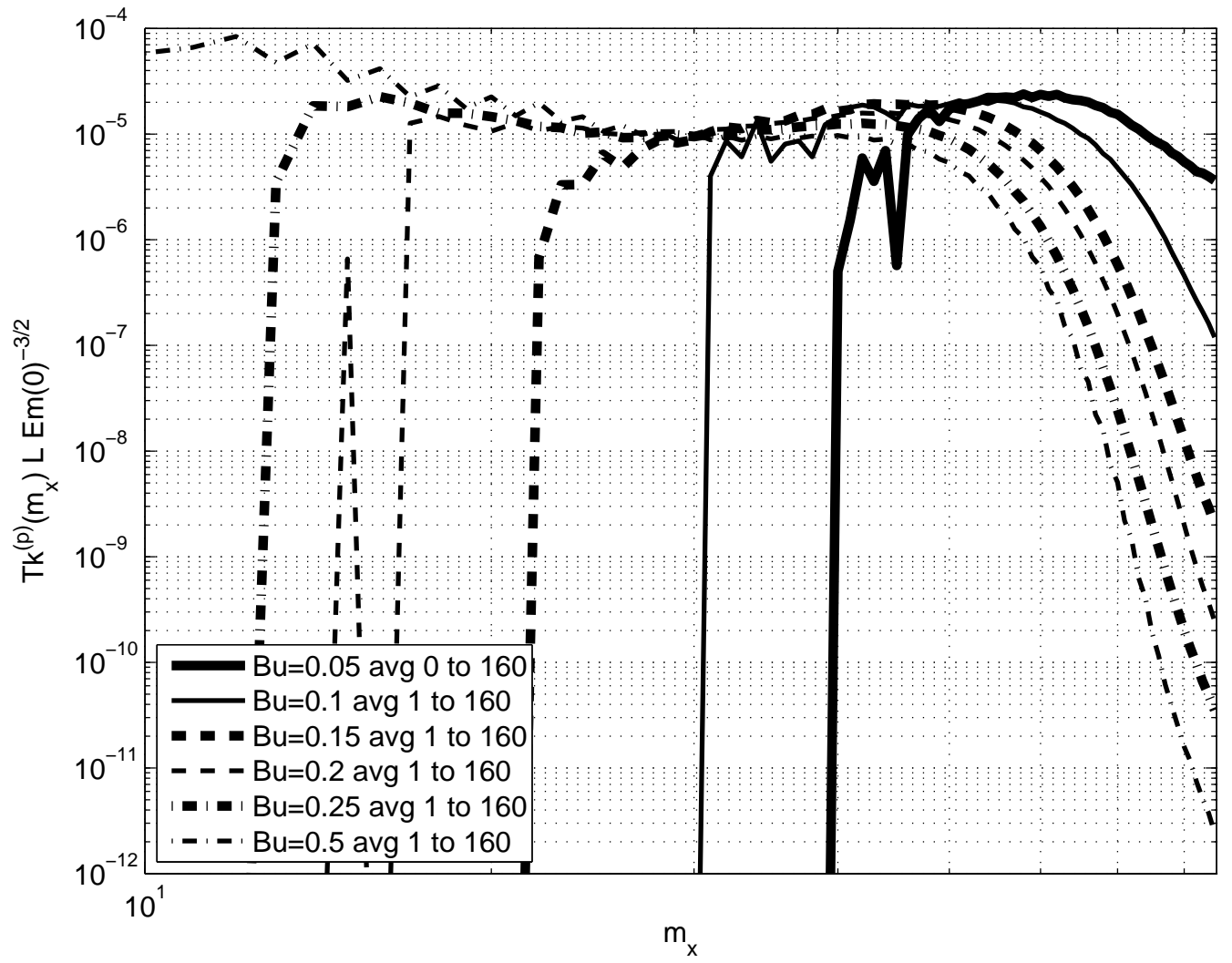

Figure 7: Downscale transfer of kinetic energy for different $B u$ for run 1, $13,22,23,24,26$ in table 1 . Averaged between $1 s$ and $160 s$ with a sampling time of $0.1 s$. 


\subsection{Energy spectra}

Given the unidirectional nature of the base flow that is being considered, a separate consideration of the $k_{x}$ and $k_{y}$ energy spectra is likely to be more informative. Figure 8 shows the kinetic energy spectra averaged over the period of the three phases of linear growth, saturation and decay as a function of $k_{x}$. As expected, the spectrum peaks at the zonal wave number of the most unstable mode in the linear phase while still being small in magnitude; $k_{x}=0$ has most of the kinetic energy. The spectra during the saturation and decay phases are seen to be similar and exhibit a slope close to $k^{-3}$. However, when plotted as a function of the horizontal wavenumber $k_{h}$ instead (see figure 9 ), the spectral fall-off is seen to be steeper, but still consistent with previous studies of geostrophic turbulence [24]. This is consistent with Charney [25] QG turbulence theory who predict a slope in -3 . We note here, that surface quasi-geostrophic (SQG) [26, 27, 28, 29] effects and other ageostrophic effect $[30,31,32]$ are expected to lead to shallower slopes. Finally, although a level of horizontal anisotropy persists even at a late stage of the evolution at most scales (figure 10 shows the kinetic energy spectra in the decay phase as functions of either the zonal or meridional wavenumbers), it is likely that this anisotropy is related to the highly anisotropic nature of the barotropic 
component of the flow (see figure 11). We do not known why there is so little barotropic energy for $m_{x}>1$ but that is a remaining observation. 


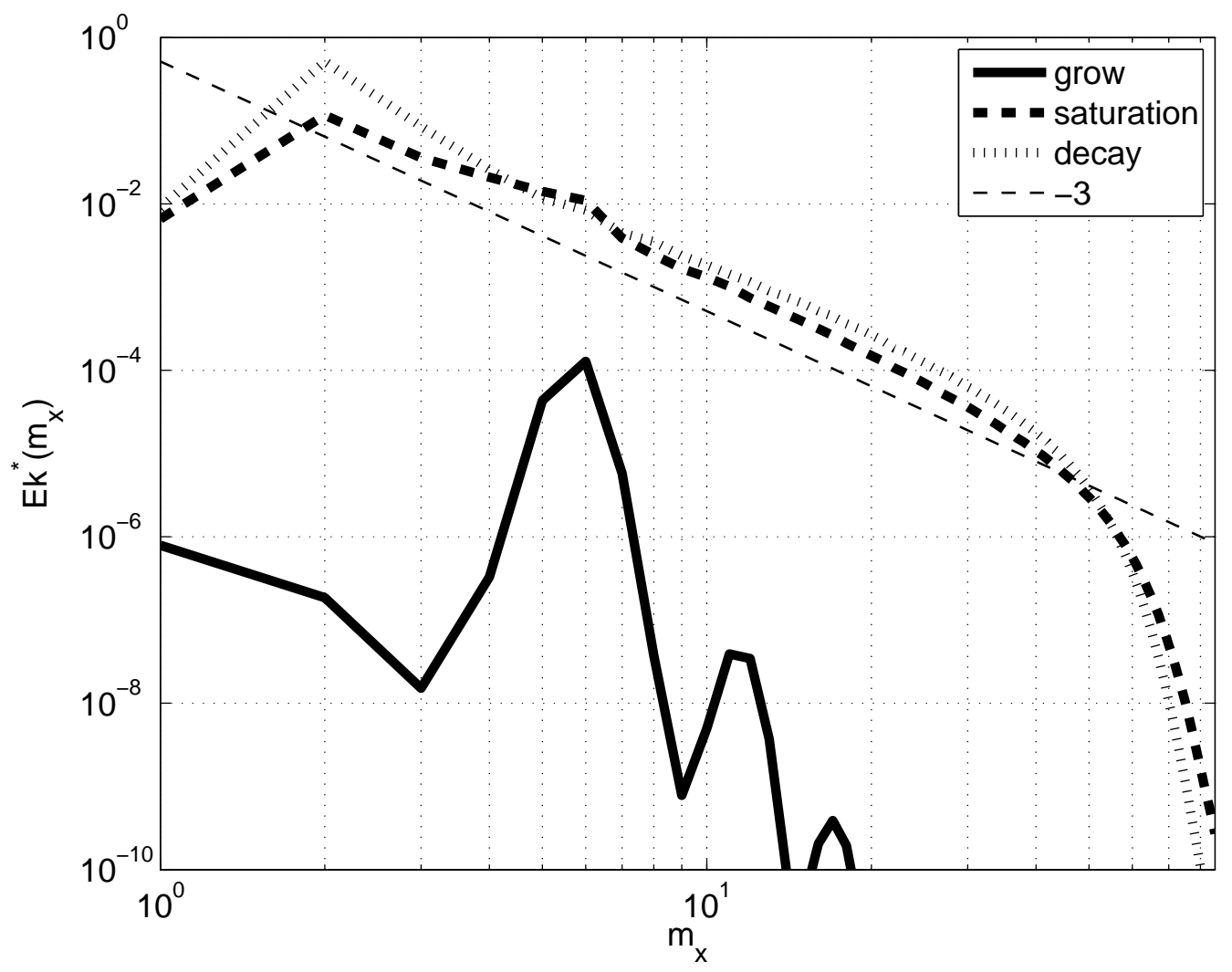

Figure 8: $k_{x}$ Kinetic energy spectra for run 13 in table 1 averaged over the three phases. Linear phase: averaged between $0 s$ and $28 s$, saturation phase: averaged between $28 s$ and $40 s$ and decay phase: averaged between $40 s$ and $160 s$ with a sampling time of $0.1 s$. 


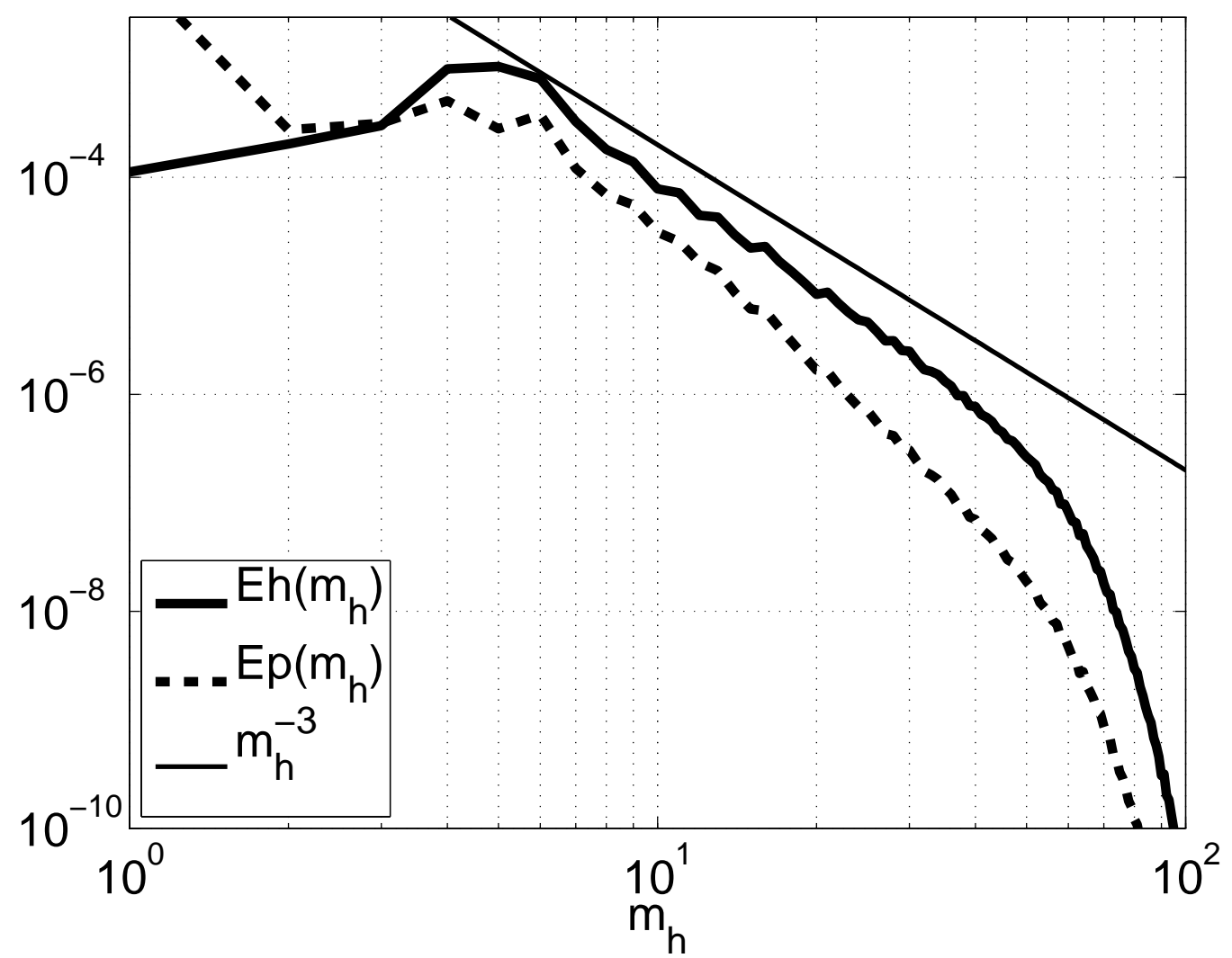

Figure 9: Potential energy and horizontal kinetic energy spectra for run 13 in table 1 during the saturation phase: averaged between $28 s$ and $40 s$ with a sampling time of $0.1 \mathrm{~s}$. 


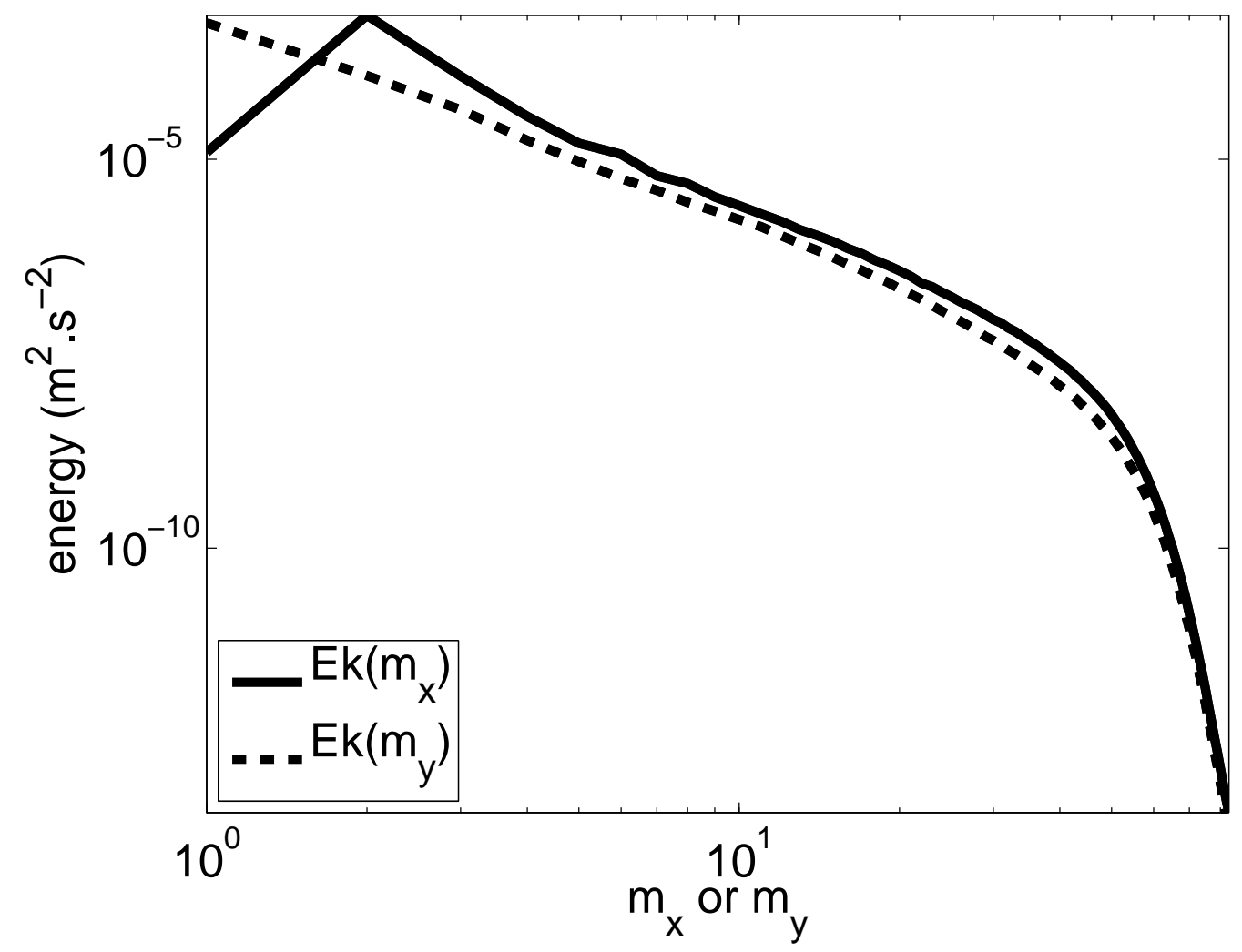

Figure 10: Kinetic energy spectra for run 13 in table 1 during the decay phase: averaged between $40 s$ and $160 s$ with a sampling time of $0.1 s$ 


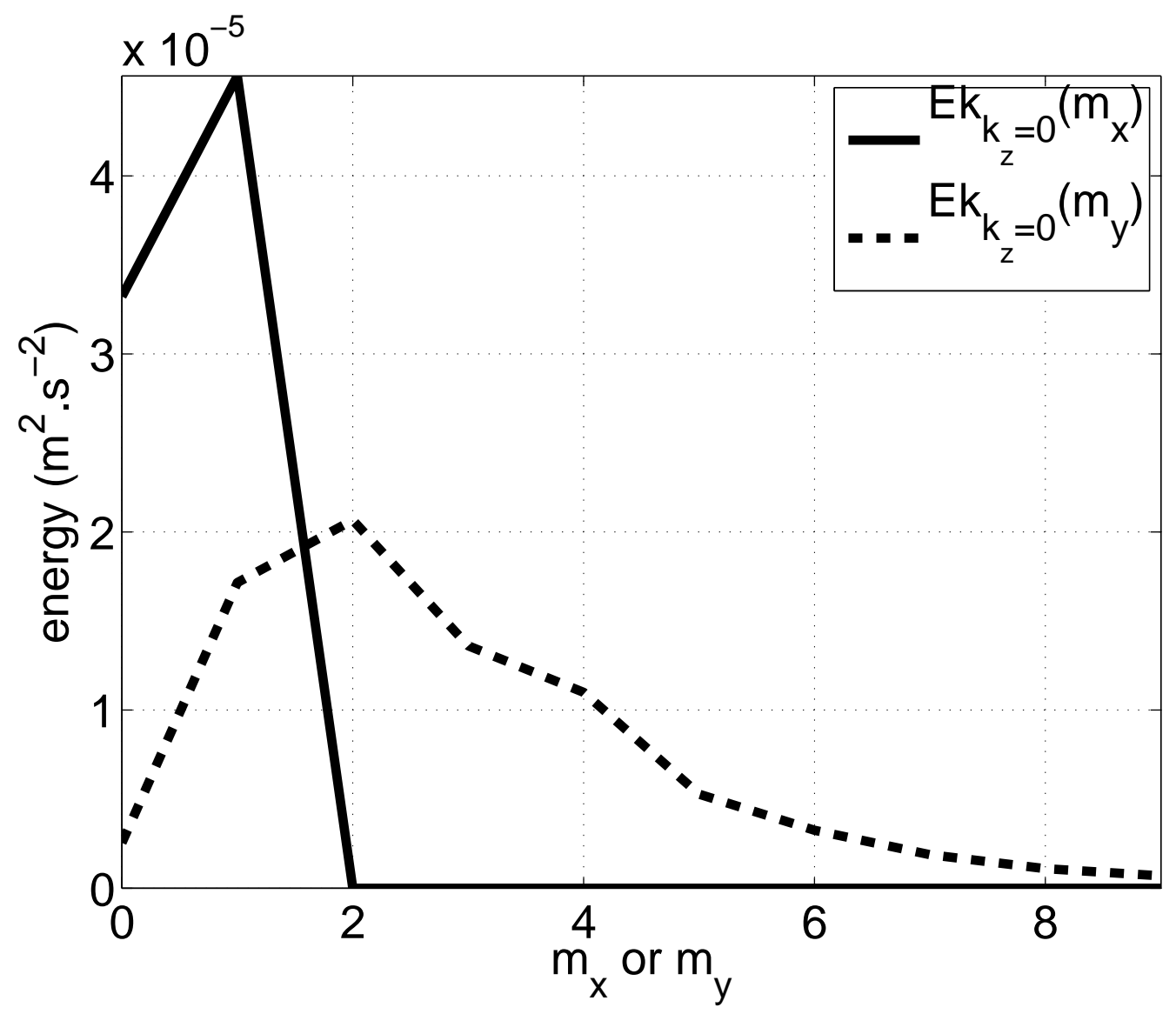

Figure 11: Barotropic kinetic energy spectra for run 13 in table 1 during the decay phase: averaged between $40 s$ and $160 s$ with a sampling time of 0.1 . 


\section{Small scale instability and Imbalance}

For $B u=0.05$ we observe a small scale instability that initiates in a weakly stratified region (see figure 13). Figures 15 is a zoom on an anticyclonic region. For deduce the cyclonicity we can look at the zonal velocity on figure 14a. The velocity in the red region is pointing toward $+x$ and the velocity in the blue region is pointing toward $-x$ meaning the vorticity will be negative hence anticyclonic since $f>0$. Further, the configuration of the flow is such that this initiation region is also anti-cyclonic - see figures $14 \mathrm{~b}$. At saturation, however, the instability is seen in the full water column (see figure 15). In the figure 16 with a different base state we can see at the same time the small scale instability and the large scale instability.

While submesoscale baroclinic instabilities grow by drawing energy from the buoyancy field [33, 34], other small scale instabilities have been identified that extract energy from the kinetic energy of the base flow [35]. Because of the configuration of the stratification and the pattern of vertical velocity, this instability is closer to latter kind. Further, localization of the instability in the region of maximum horizontal shear and minimum vertical shear suggests that this instabillity may be driven by horizontal shear. In a horizontally sheared rotating and stratified Couette flow, Vanneste and Yavneh, 
2007 [36] show that the flow is unconditionally unstable due to a resonance process involving Kelvin waves and inerto-gravity waves. The waves number of the instability is of the order of the ratio between the horizontal shear and the Coriolis frequency, and is therefore large. In McWilliams et al. (1998) [37], a condition for loss of integrability for balanced dynamics implicates what has, in a moderately extensive literature, come to be called anticyclonic ageostrophic instability (AAI), of which [36] is a small part. Vanneste and Yavneh [36] study an ageostrophic instability in a horizontal Couette flow. The initiation of the instability in an anti-cyclonic region have some similarity with Vanneste and Yavneh [36] but some important difference. Here the boundary conditions and mean flows do not support the existence of Kelvin waves, so the instability in Vanneste and Yavneh [36] is not likely related to the small-scale instability here. This small scale instability is effective in dissipating energy: For the runs 1 and 1 in table 1 with $B u=0.05$ and 0.1 respectively, the value of the small scale dissipation of kinetic energy is about 300 times greater for this instability than for the large scale instability. 


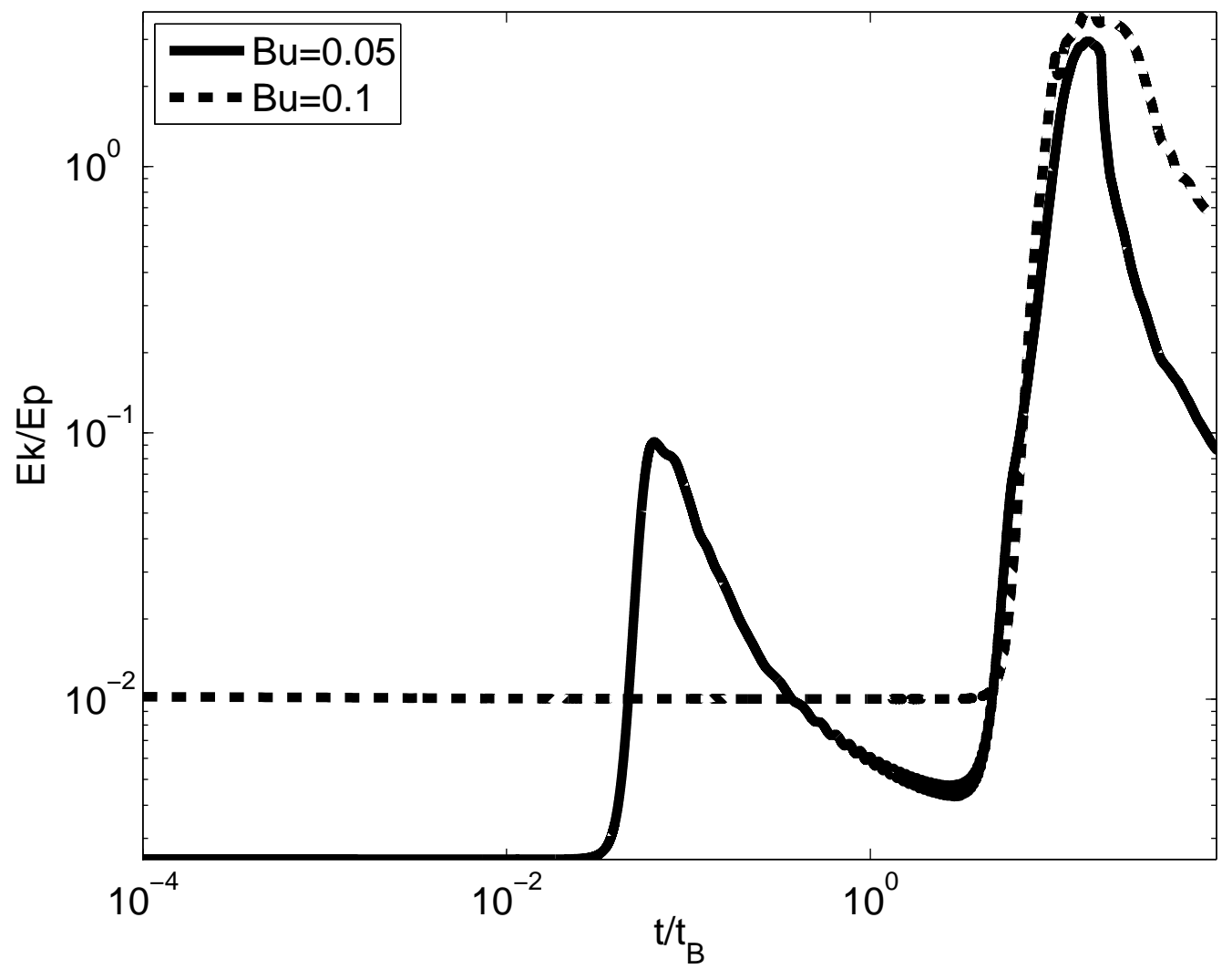

Figure 12: Evolution of the ratio between kinetic and potential energy for runs 1 and 11 in table 1 as function of the ratio of the time to the baroclinic time of the base state. 


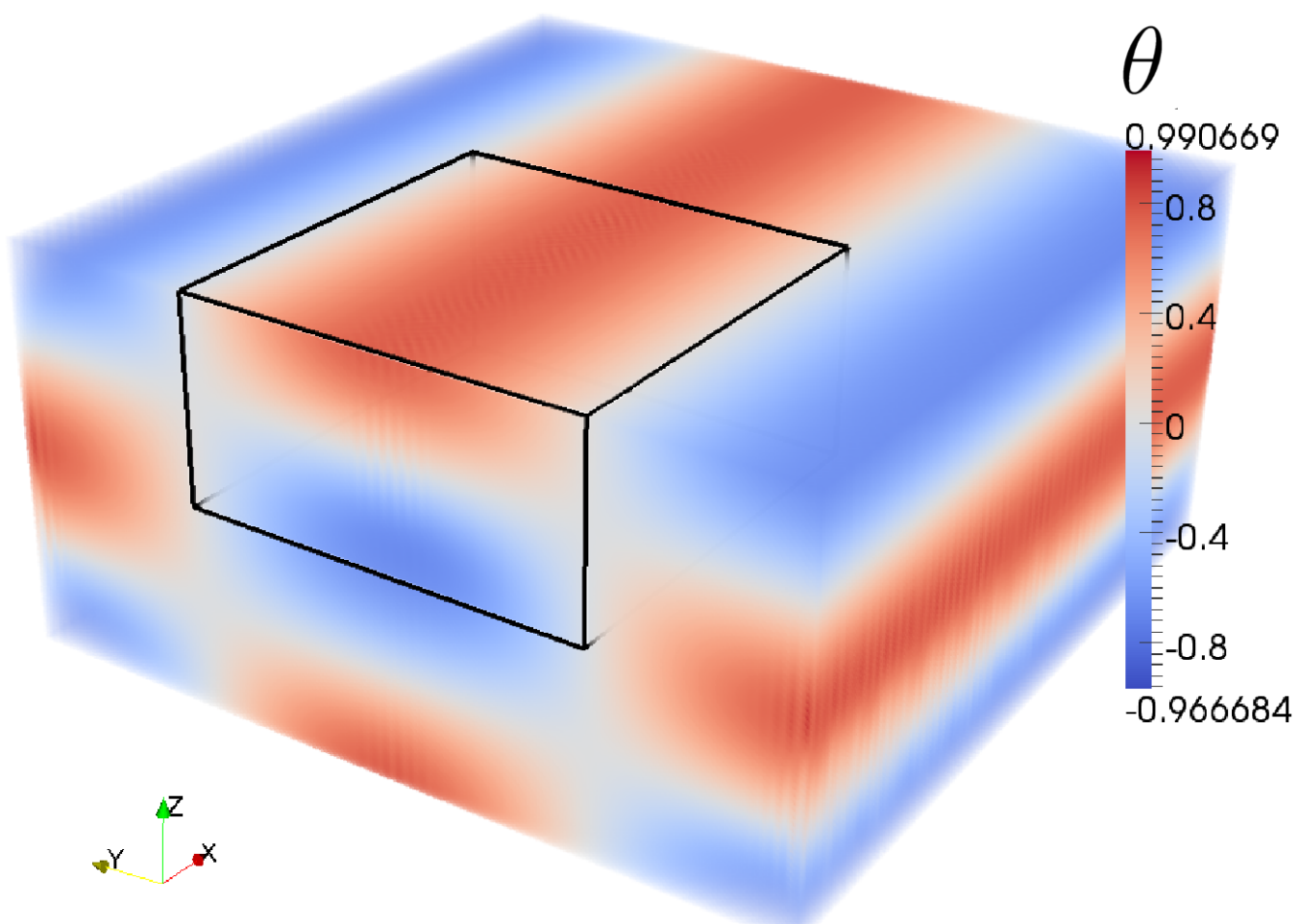

Figure 13: Perturbation Density field visualized by volume rendering and magnification for run 1 in table 1 at $t=0.1$. The black rectangle indicate the position of the figures 14d. Visualization done with ParaView [38]. 

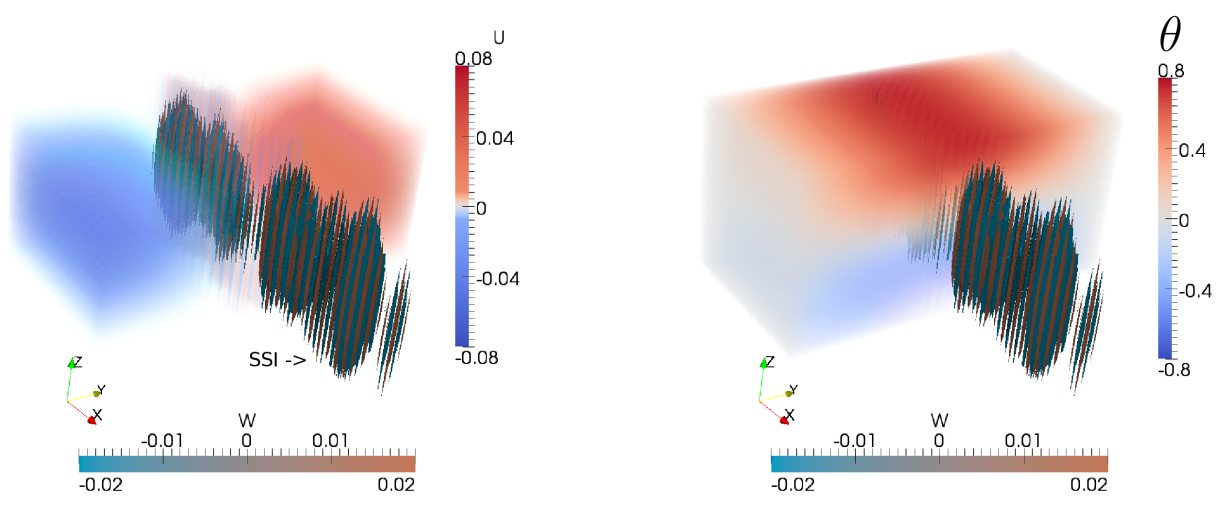

(a) $u$ at $t=0.1$

(b) $\theta$ at $t=0.1$

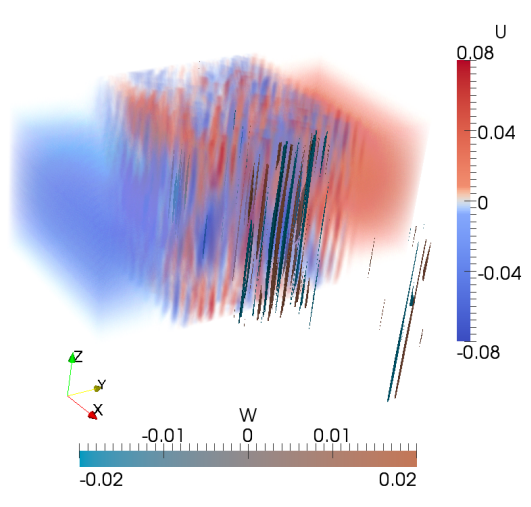

(c) $u$ at $t=0.2$

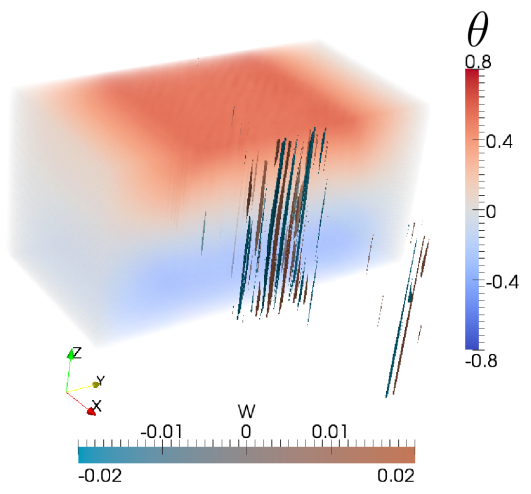

(d) $\theta$ at $t=0.2$

Figure 14: Zoom of the figure 13. Iso- $w= \pm 0.02$ and zonal velocity field (right) or perturbation density field (left) in the background visualized by volume rendering for run 1 in table 1. The acronym SSI is for Small Scall Instability and indicate the location where small scall instabilities are visible. Visualization done with ParaView [38]. 


\subsection{Inertio-gravity wave activity}

The frequency of interia gravity waves in the Boussinesq system is given by

$$
\hat{\sigma}(\mathbf{k})=\frac{\sqrt{f^{2} k_{z}^{2}+N^{2} k_{h}^{2}}}{k}
$$

Normal modes analysis of the non-hydrostatic Boussinesq equation, for $k_{h} \neq$ 0 and $k_{z} \neq 0$ leads to spectral amplitude for the waves modes as:

$$
\hat{w}^{( \pm)}=\frac{\imath k}{\sqrt{2} k_{z}}\left(k_{x} \hat{u}+k_{y} \hat{v}\right) \pm \frac{f k_{z}}{\sqrt{2} \hat{\sigma} k}\left(k_{x} \hat{v}-k_{y} \hat{u}\right) \pm \frac{N k_{h}}{\sqrt{2} \hat{\sigma} k} k_{h} \hat{\theta}
$$

The first term of equation 41 is the contribution of the horizontal divergence, the second term is the contribution of the gradient of vertical vorticity and the last term is the contribution of the horizontal perturbation density gradient. From equation 41, energy in the wave modes can be computed as:

$$
\operatorname{Ewaves}(t)=\sum_{k} \frac{1}{k_{h}^{2}}\left(\hat{w}^{(+)}(\mathbf{k}, t) \hat{w}^{(+)}(\mathbf{k}, t)^{*}+\hat{w}^{(-)}(\mathbf{k}, t) \hat{w}^{(-)}(\mathbf{k}, t)^{*}\right)
$$

The decomposition of horizontal kinetic energy into wave and vortex components, presented in figure 17 and figure 18 involve the use of the eigenvector of the normal mode decomposition.

We note that the maximum of wave energy in run 1 occurs during the initial smaller scales instability rather than during the large scale instability. 


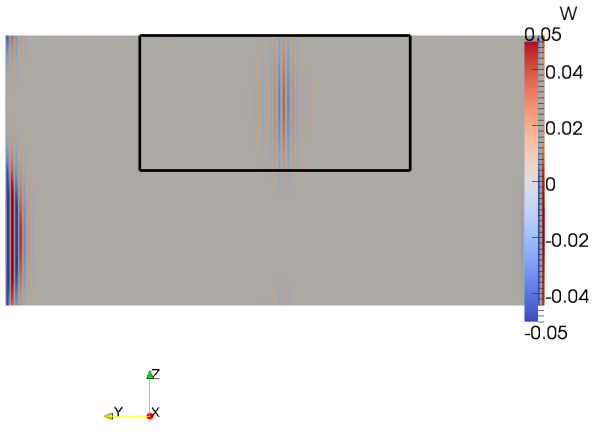

(a) $w$ at $t=0.1$

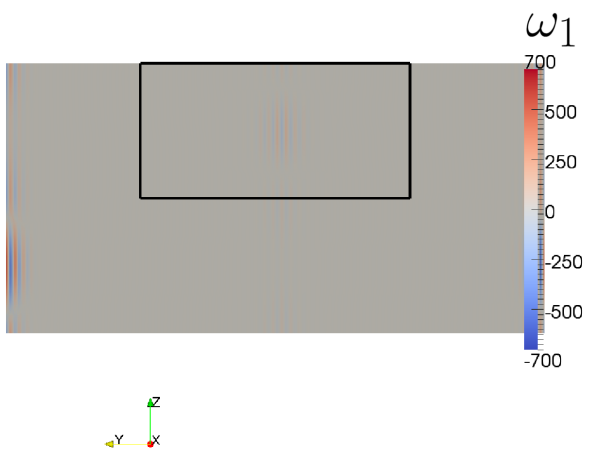

(c) $\omega_{1}$ at $t=0.1$

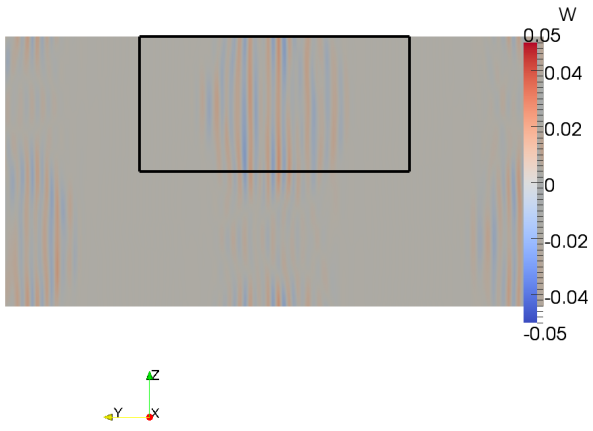

(b) $w$ at $t=0.2$

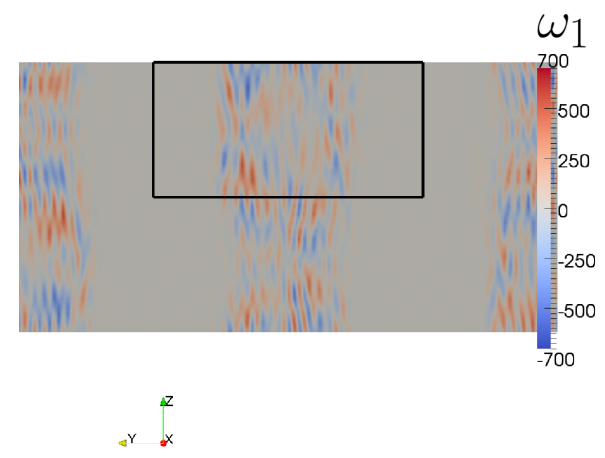

(d) $\omega_{1}$ at $t=0.2$

Figure 15: Vertical velocity field and zonal vorticity field in plane $x=0$ for run 1 in table 1 . The black rectangle is the magnification zone of figure 14d. Visualization done with ParaView [38]. 


$$
\begin{array}{lll}
\theta_{-0.4} & & \\
-0.2 \quad 0 & 0.2 & 0.4 \\
-0.57143 & 0.569367
\end{array}
$$

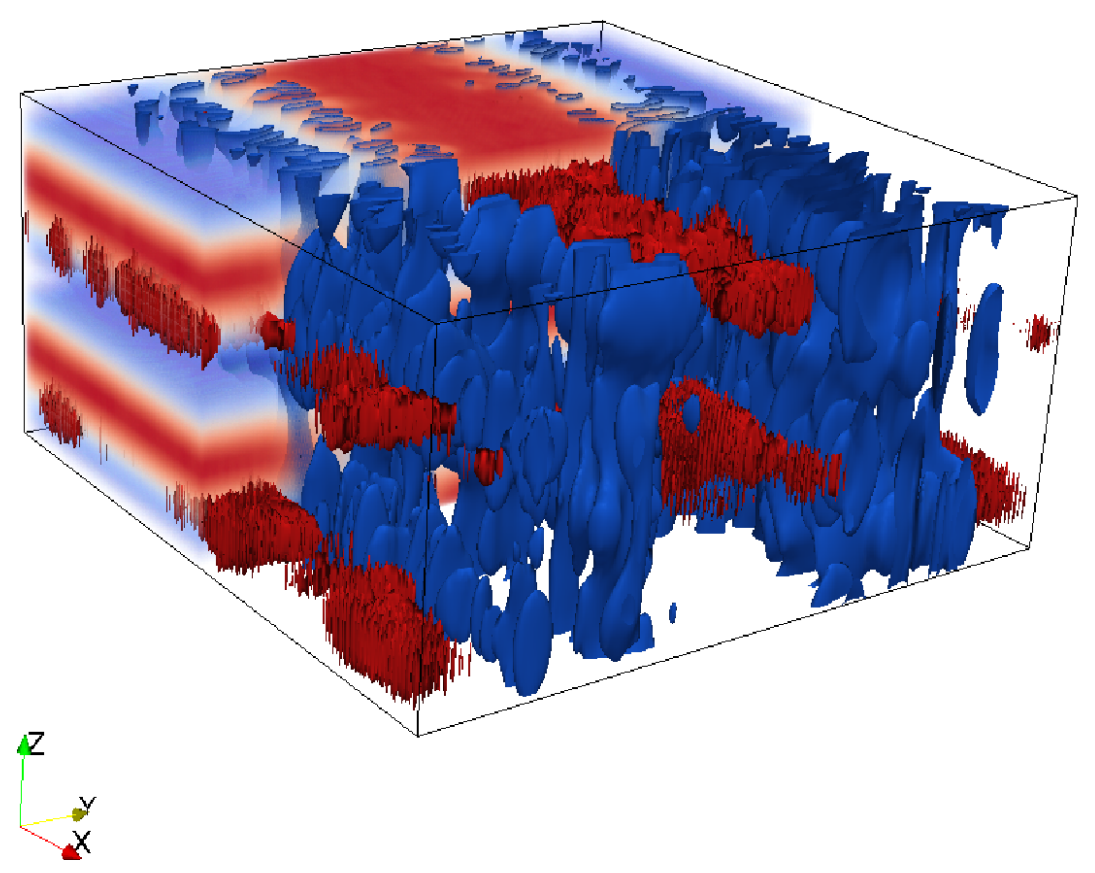

Figure 16: Surfaces in red localize the small scale instability and are isosurface $w= \pm 0.01$ of vertical velocity close to $y=0$ and $y=0.5$. Surface in blue localize the large scale instability and are isosurface $v= \pm 0.1$ of meridional velocity close to $y=0.25$ and $y=0.75$. The perturbation density field is in the background. The data are from run 2 in table 1 at $t=7 \mathrm{~s}$. Visualization done with ParaView [38]. 
Further, the imbalance generated during the small scale instability is 300 greater than that generated by the baroclinic instability $\left(E m_{\text {waves }}(0.14)=\right.$ $0.027, E h_{\text {waves }}(0.14)=0.020, E m_{\text {waves }}(10)=8.410^{-5}$ and $E h_{\text {waves }}(10)=$ $4.210^{-5}$ ). This similarity between the ratio of imbalances in the small scale and large scale instabilities and the ratio of (small-scale) dissipation associated with the small-scale and large-scale instabilities suggests that the dissipation is related to imbalanced motions. Finally, we note that wave energy associated with the baroclinic instability (at the time when kinetic energy peaks) shows a power law dependence on the Rossby number of the base flow with an exponent of about 1.5 (see figure 19). 


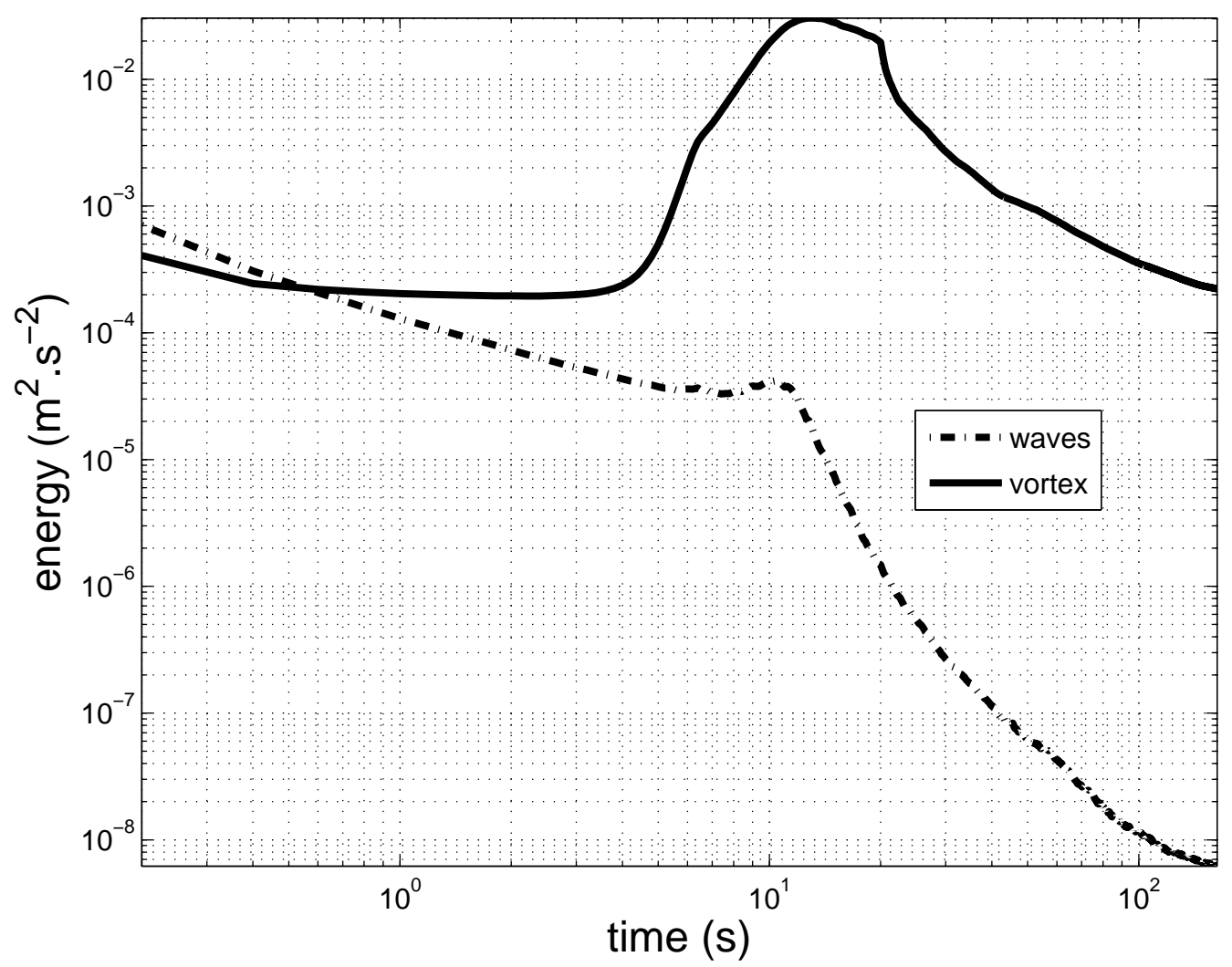

Figure 17: Contribution of wave energy and vortex energy in the horizontal kinetic energy for run 1 in table 1 


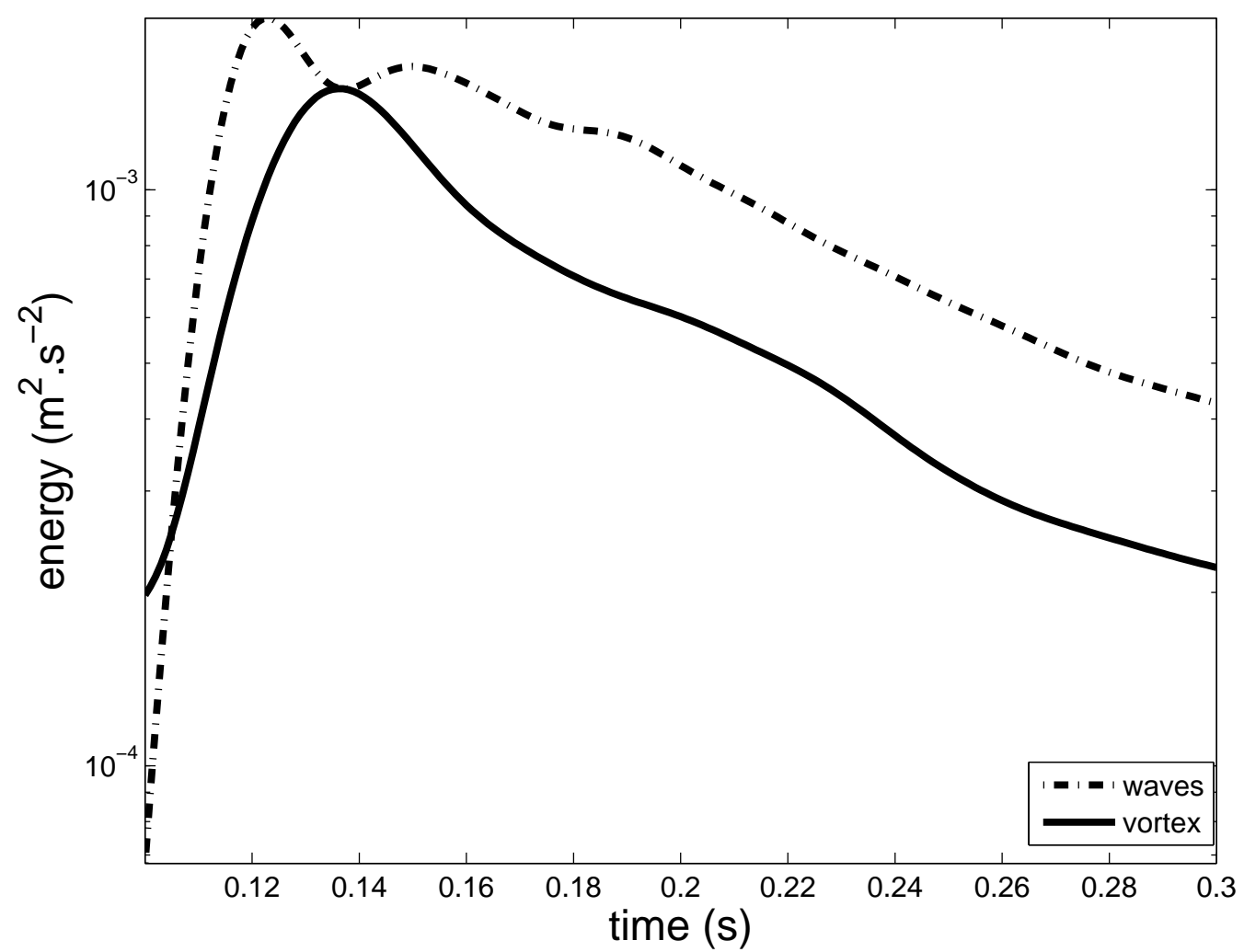

Figure 18: Contribution of wave energy and vortex energy in the horizontal kinetic energy during the period of time corresponding to the small scale instability for run 1 in table 1 


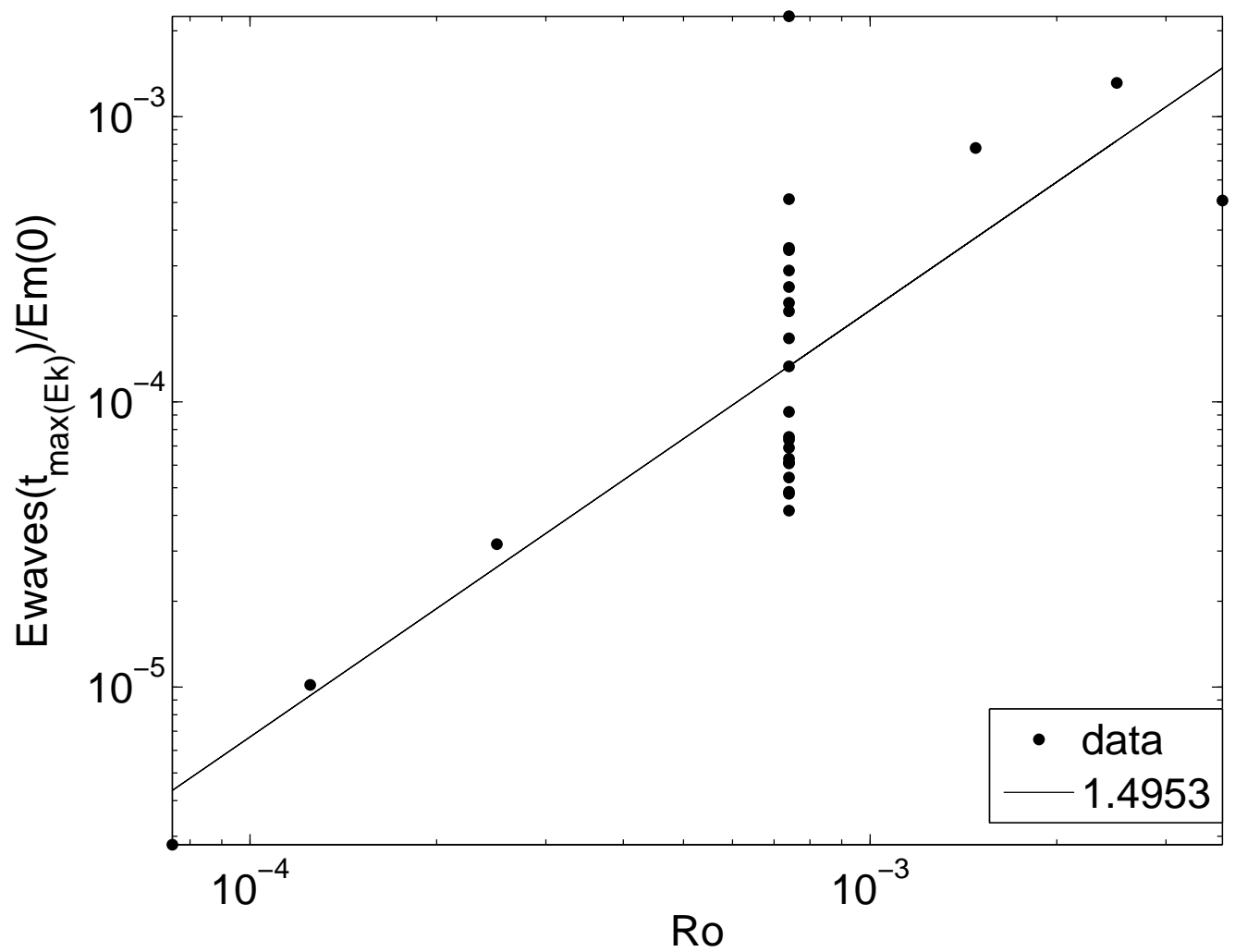

Figure 19: Kinetic energy of wave component when $E k$ is maximum as function of the Rossby number of the base state for the runs 1 to 29 in table 1 with the exception of run 17 


\subsection{Quasigeostrophic unbalanced vertical velocity field}

While there are several methods to diagnose imbalance [39], we estimate the unbalanced vertical velocity using the method of Danioux et al. [30]. For this, we define the field $\mathbf{Q}(\mathbf{x})$ as:

$$
Q_{i}(\mathbf{x})=\sum_{l=1}^{2} \partial_{i} u_{l}(\mathbf{x}) \partial_{l} \theta(\mathbf{x}), \forall i \text { in } 1 \text { or } 2
$$

and recover the spectral amplitude of the vertical QG "balanced" velocity using the $\Omega$-equation:

$$
\hat{w}^{(\Omega)}(\mathbf{k})=\frac{-2}{N} \frac{\imath \mathbf{k}_{h} \hat{\mathbf{Q}}}{k_{h}^{2}+\frac{f^{2}}{N^{2}} k_{z}^{2}}
$$

The unbalanced component is:

$$
w^{(u)}=w-w^{(\Omega)}
$$

And the unbalanced vertical kinetic energy is defined by:

$$
E w_{U}(t)=\frac{1}{2} \sum_{\mathbf{k}} \hat{w}^{(u)}(\mathbf{k}) \hat{w}^{(u)}(\mathbf{k})^{*}
$$

During the initial linear development of the instability, imbalance is seen to be localized meridionally at the center of the jet, where the horizontal shear is minimum and vertically where the vertical shear is maximum (see figures 20 and 21). The vertical velocity field obtained by the $\Omega$ equation reveal 
structure finer than the one of the vertical velocity field (see figures 20 and 21).

In figure 22 unbalanced vertical kinetic energy normalized by the initial mechanical energy at the time of the maximum of kinetic energy is plotted as a function of Rossby number. Like in the previous section 5.1 we find an power-law dependence on Rossby number with an exponent of about 1.5. The vertical kinetic energy is also ploted in 22, and the two energies are seen to be close suggesting that vertical kinetic energy in the range of parameter considered can be a good approximation of the level of imbalance in the flow.

Both the normal-mode decomposition and the omega equation equation do not appear to make an important distinction. The normal-mode decomposition is purely linear and merely describes all ageostrophic motion. This decompostion does not distinguish between unbalanced flow and higher-order corrections to quasigeostrophy. The Omega equation decompostion does. This suggest that a linear theory may be able in average to model such imbalance. 


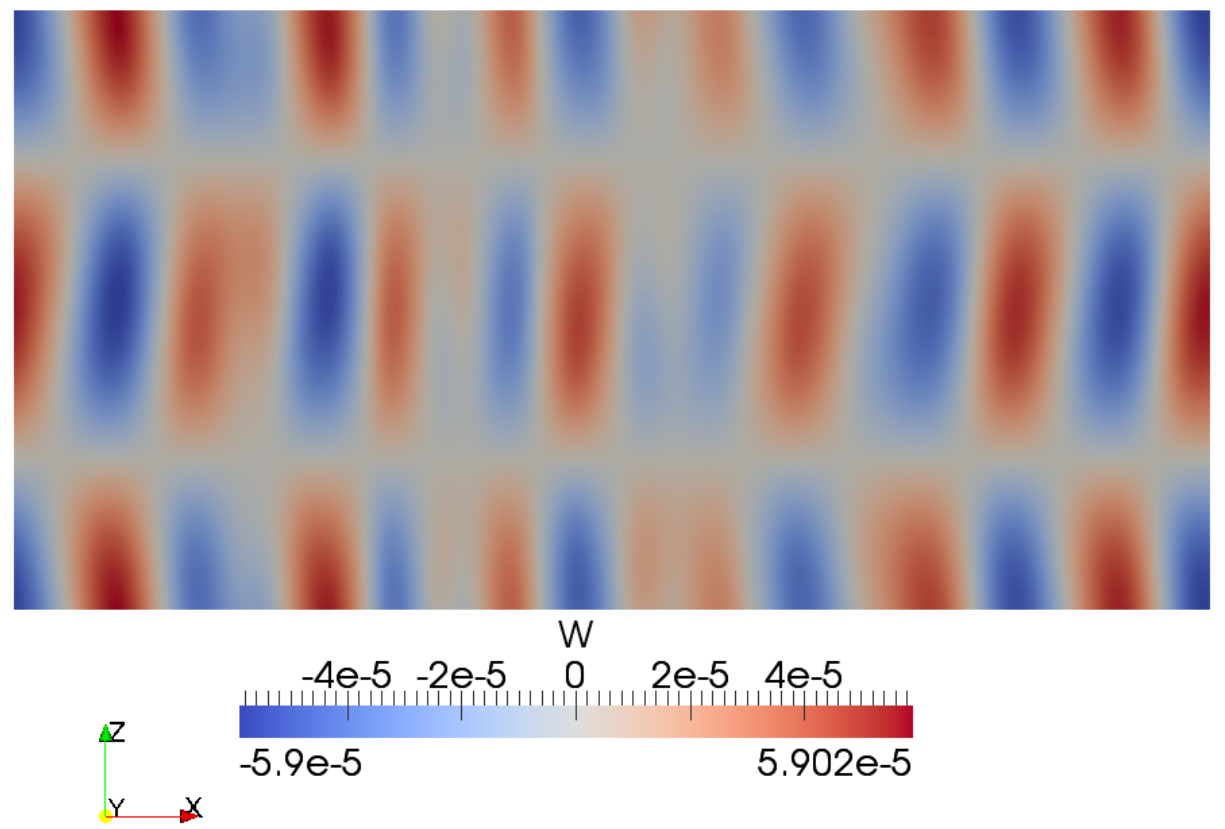

Figure 20: Vertical velocity field in $y=0$ for run 19 in table 1 during the linear growth phase at $t / \tau_{B}=4.6(B u=0.1)$. Visualization done with ParaView [38]. 

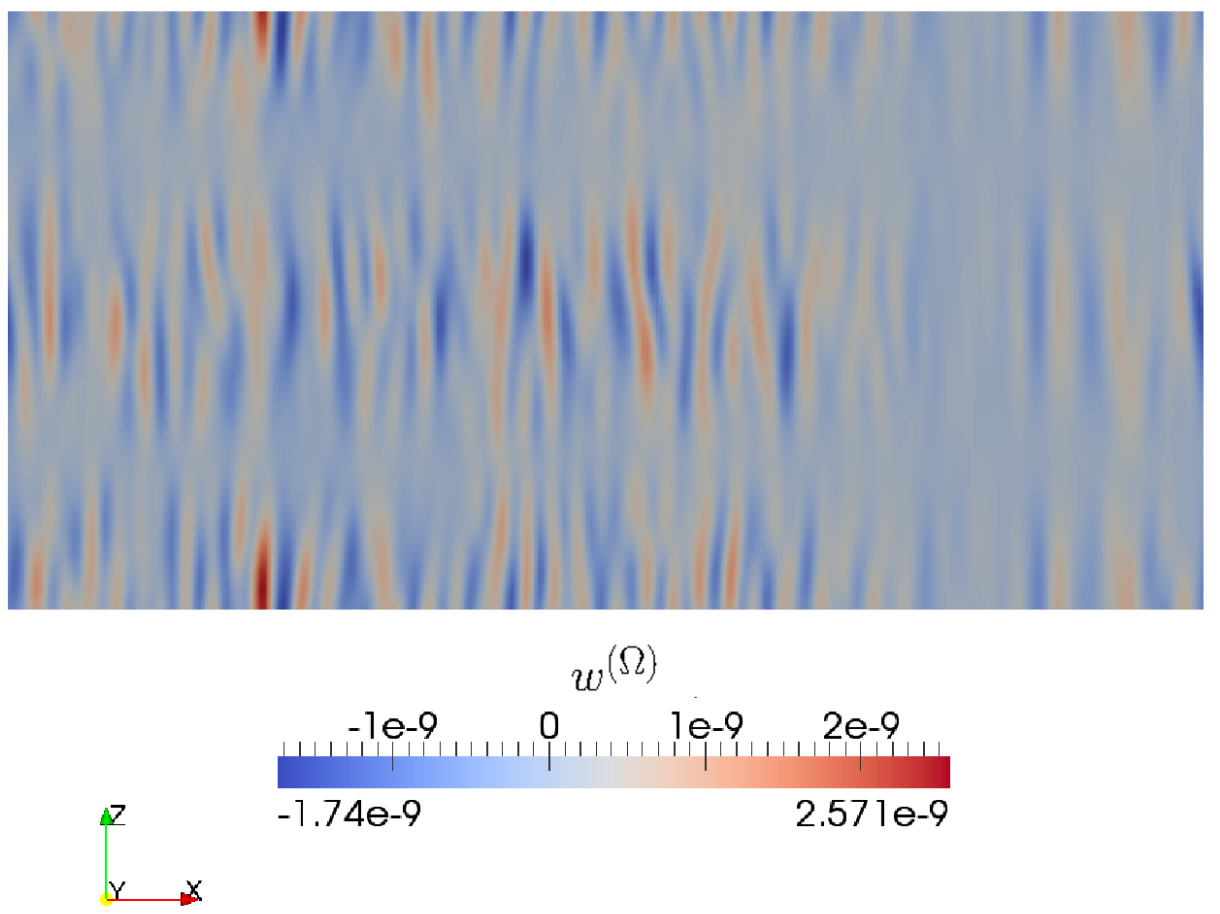

Figure 21: Vertical velocity field otained by the $\Omega$ equation at $y=0$ for run 19 in table 1 during the linear grow at $t / \tau_{B}=4.6(B u=0.1)$. Visualization done with ParaView [38]. 


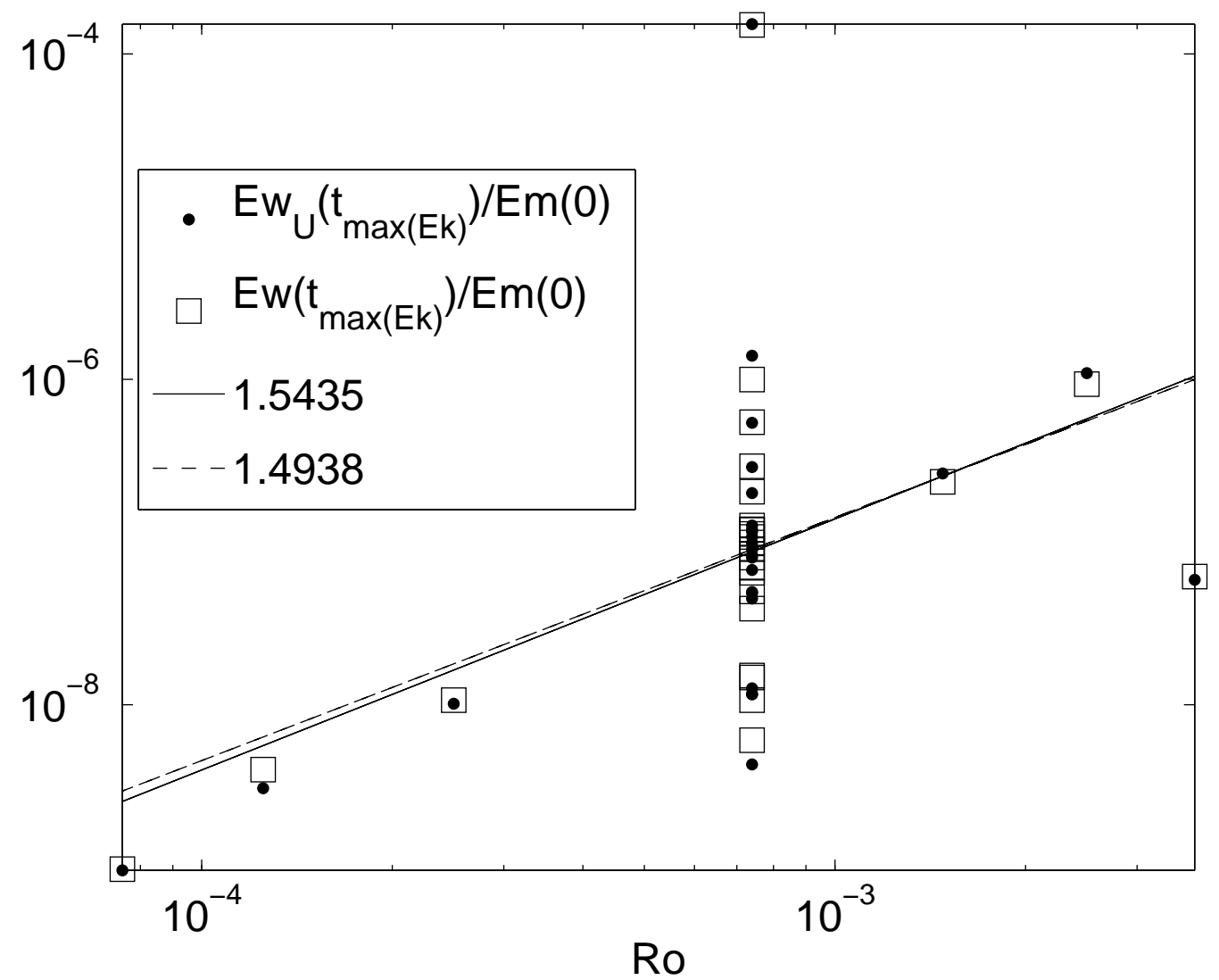

Figure 22: Unbalanced vertical kinetic energy when $E k$ is maximum (dot) and vertical kinetic energy when $E k$ is maximum (square) as function of initial Rossby number from runs 1 to 29 in table 1. 


\section{Conclusion}

In this study of rotating stratified flows, we considered the instability of a sinusoidal thermal-wind. Using fully nonlinear numerical simulations, we find first identify a large-scale, geostrophic, hydrostatic, baroclinic instability that is consistent with classical theory. We also find a small scale instability at small Burger numbers that is initiated in a weakly-stratified anti-cyclonic region. This small scale instability is likely imbalanced and results in small scale dissipation that is greater than due to the large scale instability: We quantified imbalance by considering both, a wave-vortex decomposition and the quasi-geostrophic $\Omega$-equation. Kinetic energy-spectral slopes close to -3 and consistent with quasi-geostrophic turbulence is recovered in all the simulations considered. The fact that no break in slope at high wavenumbers was observed is likely related to the limited range of scales in the simulations considered or that the Ro values are small: in decaying solutions as here, the interval of spectrum flattening may be brief [35].

\section{Acknowledgement}

This research was supported by the Laboratory Directed Research and Development (LDRD) program at Los Alamos National Laboratory (project 
number 20110150ER). Computational resources were provided by Institutional Computing at the Los Alamos National Laboratory.

[1] Norman A. Phillips. Energy Transformations and Meridional Circulations associated with simple Baroclinic Waves in a two-level, Quasigeostrophic Model. Tellus, 6(3):273-286, August 1954.

[2] K. S. Smith. The geography of linear baroclinic instability in Earth's oceans. Journal of Marine Research, 65(5):655-683, September 2007.

[3] C. E. Leith and R. H. Kraichnan. Predictability of Turbulent Flows. Journal of Atmospheric Sciences, 29(6):1041-1058, 1972.

[4] W. C. Skamarock. Evaluating Mesoscale NWP Models Using Kinetic Energy Spectra. Monthly Weather Review, 132(12):3019-3032, December 2004 .

[5] A. Vallgren, E. Deusebio, and E. Lindborg. Possible Explanation of the Atmospheric Kinetic and Potential Energy Spectra. Physical Review Letters, 107(26):1-4, December 2011.

[6] L. Bierdel, P. Friederichs, and S. Bentzien. Spatial kinetic energy spec- 
tra in the convection-permitting limited-area NWP model COSMO-DE. Meteorologische Zeitschrift, 21(3):245-258, June 2012.

[7] K. Ngan and G. E. Eperon. Middle atmosphere predictability in a numerical weather prediction model: revisiting the inverse error cascade. Quarterly Journal of the Royal Meteorological Society, 138(666):13661378, July 2012.

[8] C. Talbot, E. Bou-Zeid, and J. Smith. Nested Mesoscale Large-Eddy Simulations with WRF: Performance in Real Test Cases. Journal of Hydrometeorology, 13(5):1421-1441, October 2012.

[9] E. T. Eady. Long Waves and Cyclone Waves. Tellus, 1(3):33-52, August 1949.

[10] Guillaume Simon. Dynamique multi-échelle en fluide stratifié tournant, instabilité de cisaillement et cyclone intense. Ph.D. dissertation, École Centrale de Lyon, 2007.

[11] A. B. Pieri, C. Cambon, F. S. Godeferd, and A. Salhi. Linearized potential vorticity mode and its role in transition to baroclinic instability. Physics of Fluids, 24(7):076603, 2012. 
[12] Alexandre B. Pieri, F. S. Godeferd, C. Cambon, and A. Salhi. Nongeostrophic instabilities of an equilibrium baroclinic state. Journal of Fluid Mechanics, 734:535-566, October 2013.

[13] Aziz Salhi and Claude Cambon. Advances in Rapid Distortion Theory: From Rotating Shear Flows to the Baroclinic Instability. Journal of Applied Mechanics, 73(3):449, 2006.

[14] G. R. Mamatsashvili, V. S. Avsarkisov, G. D. Chagelishvili, R. G. Chanishvili, and M. V. Kalashnik. Transient Dynamics of Nonsymmetric Perturbations versus Symmetric Instability in Baroclinic Zonal Shear Flows. Journal of the Atmospheric Sciences, 67(9):2972-2989, September 2010 .

[15] S Rogallo and R S Rogallo. Numerical Experiments in Homogeneous Turbulence. Technical Report 81315, NASA, Moffett Field, CA, U.S.A., 1981.

[16] J. G. Charney and M. E. Stern. On the stability of internal baroclinic jets in a rotating atmosphere. Journal of Atmospheric Sciences, 19:159$172,1962$.

[17] M. A. Taylor, S. Kurien, and G. L. Eyink. Direct observation of the 
intermittency of intense vorticity filaments in turbulence. Phys. Rev. E, 68(2):26310, 2003.

[18] S. Kurien and M. A. Taylor. Direct numerical simulation of turbulence: data generation and statistical analysis. Los Alamos Science, (29):142$151,2005$.

[19] James C. McWilliams and Peter R. Gent. Intermediate Models of Planetary Circulations in the Atmosphere and Ocean. Journal of the Atmospheric Sciences, 37(8):1657-1678, August 1980.

[20] J. Holton. An Introduction to Dynamic Meteorology. Elsevier Academic Press, 4 edition, 2004.

[21] J. G. Charney. THE DYNAMICS OF LONG WAVES IN A BAROCLINIC WESTERLY CURRENT. Journal of Meteorology, 4(5):136162, October 1947.

[22] J. Pedlosky. Geophysical Fluid Dynamics. Springer Study Edition. Springer-Verlag, second edition, 1987.

[23] J. C. McWilliams. Fundamentals of Geophysical Fluid Dynamics, volume 576. Cambridge University Press, March 2007. 
[24] K. S. Smith and G. K. Vallis. The Scales and Equilibration of Midocean Eddies: Forced-Dissipative Flow. Journal of Physical Oceanography, 32(6):1699-1720, June 2002.

[25] J. G. Charney. Geostrophic turbulence. Journal of the Atmospheric Sciences, 28(108):95, 1971.

[26] I. M. Held, R. T. Pierrehumbert, S. T. Garner, and K. L. Swanson. Surface quasi-geostrophic dynamics. Journal of Fluid Mechanics, 282(1):1, April 2006 .

[27] P. Klein, B. L. Hua, G. Lapeyre, X. Capet, S. Le Gentil, and H. Sasaki. Upper Ocean Turbulence from High-Resolution 3D Simulations. Journal of Physical Oceanography, 38(8):1748, 2008.

[28] P. Klein, G. Lapeyre, G. Roullet, S. Le Gentil, and H. Sasaki. Ocean turbulence at meso and submesoscales: connection between surface and interior dynamics. Geophysical \&3 Astrophysical Fluid Dynamics, 105(45):421-437, August 2011.

[29] H. Sasaki and P. Klein. SSH Wavenumber Spectra in the North Pacific from a High-Resolution Realistic Simulation. Journal of Physical Oceanography, 42(7):1233-1241, July 2012. 
[30] E. Danioux, J. Vanneste, P. Klein, and H. Sasaki. Spontaneous inertiagravity-wave generation by surface-intensified turbulence. Journal of Fluid Mechanics, 699:153-173, April 2012.

[31] M. Nikurashin, G. K. Vallis, and A. Adcroft. Routes to energy dissipation for geostrophic flows in the Southern Ocean. Nature Geoscience, 6(1):48-51, December 2012.

[32] E. D. Skyllingstad and R. M. Samelson. Baroclinic Frontal Instabilities and Turbulent Mixing in the Surface Boundary Layer. Part I: Unforced Simulations. Journal of Physical Oceanography, 42(10):1701-1716, October 2012 .

[33] G. Boccaletti, R. Ferrari, and B. Fox-Kemper. Mixed Layer Instabilities and Restratification. Journal of Physical Oceanography, 37(9):22282250, September 2007.

[34] X. Capet, J. C. McWilliams, M. J. Molemaker, and A. F. Shchepetkin. Mesoscale to Submesoscale Transition in the California Current System. Part II: Frontal Processes. Journal of Physical Oceanography, 38(1):4464, January 2008.

[35] M. J. Molemaker, J. C. McWilliams, and X. Capet. Balanced and un- 
balanced routes to dissipation in an equilibrated Eady flow. Journal of Fluid Mechanics, 654:35-63, June 2010.

[36] J. Vanneste and I. Yavneh. Unbalanced instabilities of rapidly rotating stratified shear flows. Journal of Fluid Mechanics, 584:373, July 2007.

[37] James C McWilliams, Irad Yavneh, Michael J P Cullen, and Peter R Gent. The breakdown of large-scale flows in rotating, stratified fluids. Physics of Fluids (1994-present), 10(12), 1998.

[38] A. Henderson. ParaView Guide, A Parallel Visualization Application. Kitware Inc., 2007.

[39] D. G. Dritschel and L. Videz. A balanced approach to modelling rotating stably stratified geophysical flows. Journal of Fluid Mechanics, 488:123150, July 2003. 